\title{
THE MULTIPLICITY OF T TAURI STARS IN THE STAR FORMING REGIONS TAURUS-AURIGA AND OPHIUCHUS-SCORPIUS: A 2.2 MICRON SPECKLE IMAGING SURVEY
}

\author{
A. M. Ghez, ${ }^{1}$ G. Neugebauer, AND K. MatThews \\ Palomar Observatory, California Institute of Technology, Pasadena, California 91125 \\ Electronic mail: aghez@as.arizona.edu, gxn@ tacos.caltech.edu, kym@ tacos.caltech.edu \\ Received 1993 March 17; revised 1993 July 28
}

\begin{abstract}
We present the results of a magnitude limited $(K<=8.5 \mathrm{mag})$ speckle imaging survey of $69 \mathrm{~T}$ Tauri stars in the star forming regions Taurus-Auriga and Ophiuchus-Scorpius. Thirty-three companion stars were found with separations ranging from 0.07 to 2 ".5; nine are new detections. This survey reveals a distinction between the classical T Tauri stars (CTTS) and the weak-lined T Tauri stars (WTTS) based on the binary star frequency as a function of separation: the WTTS binary star distribution is enhanced at the closer separations $(<50 \mathrm{AU})$ relative to the CTTS binary star distribution. We suggest that the nearby companion stars shorten the accretion time scale in multiple star systems, thereby accounting for the presence of WTTS that are coeval with many CTTS. The binary star frequency in the projected linear separation range 16 to $252 \mathrm{AU}$ for T Tauri stars $(60[ \pm 17] \%)$ is a factor of 4 greater than that of the solar-type main-sequence stars $(16[ \pm 3] \%)$. Given the limited separation range of this survey, the rate at which binaries are detected suggests that most, if not all, $T$ Tauri stars have companions. We propose that the observed overabundance of companions of $\mathrm{T}$ Tauri stars is an evolutionary effect, in which triple and higher order $\mathrm{T}$ Tauri stars are disrupted by close encounters with another star or system of stars.
\end{abstract}

\section{INTRODUCTION}

T Tauri stars are a class of young, low mass (0.1 to 3.0 $\mathscr{M}_{\odot}$ ), pre-main-sequence stars whose ages are in the range of $10^{4}-10^{7} \mathrm{yr}$. Most evolutionary theories of how T Tauri stars emerge from the deeply embedded protostellar stage and progress toward the main sequence are based on single star scenarios (e.g., Adams et al. 1988); surveys of mainsequence stars, however, have already shown that about $2 / 3$ of the solar-type stars are in multiple star systems (Abt \& Levy 1976; Abt 1983; Duquennoy \& Mayor 1991). The question therefore arises of how and when these multiple star systems form, for they appear to be the norm as opposed to the exception. The early work of Joy \& Van Biesbroeck (1944) showed that 5 of the first 11 known T Tauri stars are binary stars, which suggests that multiple star systems are formed early in the star formation process. Since 1944 more than $450 \mathrm{~T}$ Tauri stars have been identified (see, for example, Herbig \& Bell 1988), but only recently has the question of how many of these stars are in multiple systems been revisited.

Recent surveys, which have begun to reveal $\mathrm{T}$ Tauri companion stars, include lunar occultations (Simon 1992a; Simon et al. 1992; Leinert et al. 1991; Chen et al. 1990; Simon et al. 1987), radial velocity measurements (Mathieu 1992a; Mathieu et al. 1988), direct imaging work (Simon et al. 1992; Zinnecker et al. 1992), and speckle imaging work (Leinert et al. 1992). Each technique is sensitive to a unique limited range of binary star

${ }^{1}$ Present address: Steward Observatory, University of Arizona, Tucson, AZ 85721. separations. Thus the different surveys are complementary, since several methods of detection are necessary to deduce the fraction of young stars with companions.

This paper reports the results of a magnitude limited speckle imaging survey of $\mathrm{T}$ Tauri stars in the star forming regions Taurus-Auriga (Tau-Aur) and OphiuchusScorpius (Oph-Sco). At the distances of these regions, speckle imaging is capable of detecting companion stars that are separated by less than the predicted circumstellar disk size, 100 AU (e.g., Beckwith \& Sargent 1992). Thus speckle imaging techniques permit the study of both the frequency of companion stars and their effect on a circumstellar disk.

\section{SAMPLE}

This survey was confined to the two nearest star forming regions observable from the northern hemisphere, TauAur (140 pc, Elias 1978) and Oph-Sco (140 pc for Oph, de Geus \& Burton 1991; 160 pc for Sco, de Geus et al. 1989). These regions have many similarities; in particular, they are both sites of active low mass star formation. They are, however, quite different in their distributions of molecular gas and stellar densities. Both regions have been extensively studied and a considerably body of literature on their stellar and cloud properties exists (e.g., Lada et al. 1992; Herbig \& Bell 1988, and references therein).

The compilation of Herbig \& Bell (1988) and recent X-ray selected source lists (Bouvier \& Appenzeller 1991; Walter et al. 1993) were used to identify a total of $143 \mathrm{~T}$ Tauri stars in the regions of interest. Following the example of many researchers (e.g., Walter 1986; Strom et al. 
1989), the sample was divided into classical T Tauri stars (CTTS), which have an equivalent width $W(H \alpha)>10 \AA$, and weak-lined $\mathrm{T}$ Tauri stars (WTTS), which have $W(\mathrm{H} \alpha)<10 \AA$ (the "naked T Tauri" stars defined by Walter are a subset of the WTTS).

For speckle imaging of $\mathrm{T}$ Tauri stars, the optimum bandpass is $K(2.2 \mu \mathrm{m})$ as a result of three competing effects: (1) the spectral energy distributions of $\mathrm{T}$ Tauri stars peak near $1 \mu \mathrm{m}$, (2) the coherence time of the atmosphere, and hence the exposure time used in speckle imaging, increases with wavelength as $\lambda,{ }^{1.2}$ and (3) beyond 3 $\mu \mathrm{m}$ thermal noise dominates the readout noise of present infrared arrays. The limiting magnitude of the speckle system at Palomar is $K=8.5$ mag. Thus only stars brighter than this were included, resulting in a final observing list of $95 \mathrm{~T}$ Tauri stars (66\% of the identified $\mathrm{T}$ Tauri stars). Since the $K$ magnitudes of T Tauri stars occasionally vary unpredictably, the most recent available magnitudes were used. Many of the stars in the original sample lacked nearinfrared photometry and were therefore excluded from the final observing list although they may have been bright enough to measure.

\section{OBSERVATIONS}

Over the period 1990 July to 1991 November, 69 of the $95 \mathrm{~T}$ Tauri stars were observed at the $f / 415$ Cassegrain focus of the Hale $5 \mathrm{~m}$ Telescope of Palomar Observatory using the $58 \times 62$ pixel InSb array camera in the photometric $K$ band. The pixel scale, determined by observing and reconstructing images of several binaries with well known orbits (McAlister \& Hartkopf 1988), was 0.053 $\times 0.053$ ( \pm 0 ".001). All the $19 \mathrm{~T}$ Tauri stars on the final observing list brighter than $K=7.0$ mag were observed; 26 stars with $7.0<K<=8.5 \mathrm{mag}$ remain to be measured. Table 1 (a) lists the 24 stars observed in Oph-Sco along with their positions, $K$ magnitudes, CTTS/WTTS designation, and assumed distances. Likewise, Table 1(b) presents the same information for the 45 stars observed in Tau-Aur.

Table 2 is a journal of the observations. For each source the observations proceeded in a number of object/ calibrator "pairs." A "pair" consists of a series of 400 snapshots (100 ms exposures) on the object of interest followed by a similar series of measurements on a calibrator source. Here the "calibrator" is a source expected to be unresolved by the $5 \mathrm{~m}$ telescope. Given the unprecedented angular resolution achieved, two calibrator stars were observed for each target star to ensure that at least one calibrator is an unresolved point source. Calibration of the calibrators against one another provided an adequate check that this was true. Both calibrators, if point sources, were used in the initial image reconstruction, but in most cases one calibrator is preferred due to the other being either too bright or significantly farther away from the target. Table 2 lists the calibrator star used in the final data reduction, the number of sets obtained, and the seeing at $2.2 \mu \mathrm{m}$. The FWHM of integrated calibrator data provided an estimate of the typical $2.2 \mu \mathrm{m}$ seeing during these observations, $0.85 \pm 0$ ". 23 .

TABLE 1(a). Oph-Sco sample.

\begin{tabular}{|c|c|c|c|c|c|c|c|c|c|}
\hline HBC & Name & RA (1950) & DEC (1950) & $\mathrm{K}$ (mag) & $\operatorname{Ref}^{K}$ & Type & $\operatorname{Ref}^{\text {TTS }}$ & Dist & $\operatorname{Ref}^{\text {Dist }}$ \\
\hline 254 & AS 205 & 160837.7 & -183043 & 5.8 & 5 & ctts & 1 & 160 & 8 \\
\hline 637 & DoAr 21 & 162301.7 & -241650 & 6.1 & 4 & wtts & 2 & 140 & 9 \\
\hline 649 & RNO 90 & 163117.5 & -154204 & 6.5 & 6 & ctts & 1 & 140 & 9 \\
\hline \multirow[t]{2}{*}{639} & DoAr $24 \mathrm{E}$ & 162322.0 & -241414 & 6.7 & 4 & wtts & 2 & 140 & 9 \\
\hline & ROXs 43A & 162818.1 & -242340 & 6.7 & 3 & wtts & 2,3 & 140 & 9 \\
\hline 270 & V1121 Oph & 164625.2 & -141656 & 6.8 & 5 & ctts & 1 & 140 & 9 \\
\hline 264 & SR 9 & 162438.9 & -241523 & 6.9 & 4 & ctts & 1,2 & 140 & 9 \\
\hline \multirow[t]{2}{*}{262} & SR $24 S$ & 162356.6 & -243855 & 7.1 & 7 & ctts & 1 & 140 & 9 \\
\hline & $155203-2338$ & 155202.6 & $\begin{array}{lll}-23 & 38 & 29\end{array}$ & 7.1 & 3 & wtts & 3 & 160 & 8 \\
\hline \multirow[t]{2}{*}{643} & SR 20 & 162531.2 & -241608 & 7.1 & 4 & ctts & 1,2 & 140 & 9 \\
\hline & ROXs $42 \mathrm{C}$ & 162813.6 & -242736 & 7.2 & 3 & wtts & 2,3 & 140 & 9 \\
\hline 257 & Haro 1-4 & 162210.6 & $\begin{array}{lll}-23 & 12 & 26\end{array}$ & 7.3 & 4 & ctts & 1 & 140 & 9 \\
\hline 268 & Haro 1-16 & 162831.7 & -242110 & 7.5 & 4 & ctts & 1,2 & 140 & 9 \\
\hline 634 & $160946-1851$ & 160946.4 & -185148 & 7.5 & 3 & wtts & 3 & 160 & 8 \\
\hline 259 & SR 4 & 162254.9 & -241402 & 7.5 & 4 & ctts & 1,2 & 140 & 9 \\
\hline 630 & $160815-1857$ & 160814.7 & -185702 & 7.7 & 3 & wtts & 3 & 160 & 8 \\
\hline \multirow[t]{2}{*}{267} & Haro 1-14 & 162803.9 & $\begin{array}{lll}-23 & 58 & 12\end{array}$ & 7.8 & 4 & ctts & 1 & 140 & 9 \\
\hline & $162218-2420$ & 162218.1 & -242001 & 7.8 & 3 & wtts & 3 & 140 & 9 \\
\hline \multirow[t]{2}{*}{266} & V853 Oph & 162543.7 & -242142 & 8.0 & 4 & ctts & 1,2 & 140 & 9 \\
\hline & $155913-2233$ & 155912.6 & -223309 & 8.1 & 3 & wtts & 3 & 160 & 8 \\
\hline 633 & $160905-1859$ & 160905.1 & -185912 & 8.1 & 3 & wtts & 3 & 160 & 8 \\
\hline \multirow[t]{3}{*}{638} & DoAr 24 & 162315.8 & -241336 & 8.1 & 4 & ctts & 1,2 & 140 & 9 \\
\hline & $155828-2232$ & 155827.8 & -223218 & 8.5 & 3 & wtts & 3 & 160 & 8 \\
\hline & $160827-1813$ & 160827.3 & $\begin{array}{lll}-18 & 13 & 11\end{array}$ & 8.5 & 3 & wtts & 3 & 160 & 8 \\
\hline
\end{tabular}

HBC = Catalogue entry number in Herbig \& Bell (1988)
(1) Herbig \& Bell (1988)
(4) Rydgren et al. (1976)
(2) Bouvier \& Appenzeller (1991)
(5) Glass \& Penston (1974)
(7) Graham (1991)
(3) Walter et al. (1993)
(6) Herbst \& Warner (1981)
(8) de Geus et al. (1989)
(9) de Geus \& Burton (1991) 
TABLE 1(b). Tau-Aur sample.

\begin{tabular}{|c|c|c|c|c|c|c|c|c|c|}
\hline HBC & Object & RA (1950) & DEC (1950) & K (mag) & $\operatorname{Ref}^{X}$ & Type & $\operatorname{Ref}^{\text {TTS }}$ & Dist & $\operatorname{Ref}^{\text {Dist }}$ \\
\hline 35 & T Tau & 041904.2 & +192505 & 5.3 & 6 & ctts & 1 & 140 & 11 \\
\hline 34 & RY Tau & 041850.8 & +281935 & 5.7 & 7 & ctts & 1 & 140 & 11 \\
\hline 79 & SU Aur & 045247.8 & +302919 & 6.0 & 5 & wtts & 1,14 & 140 & 11 \\
\hline 74 & DR Tau & 044413.2 & +165324 & 6.4 & 3 & ctts & 1 & 140 & 11 \\
\hline 367 & V773 Tau & 041107.3 & +280441 & 6.5 & 4 & wtts & 1 & 140 & 11 \\
\hline 37 & DG Tau & 042401.0 & +255936 & 6.7 & 3 & ctts & 1 & 140 & 11 \\
\hline 36 & DF Tau & 042359.6 & +253541 & 6.8 & 2 & ctts & 1 & 140 & 11 \\
\hline 25 & CW Tau & 041111.3 & +280327 & 6.9 & 3 & ctts & 1 & 140 & 11 \\
\hline $80 / 81$ & RW Aur & 050437.7 & +302014 & 6.9 & 2 & ctts & 1 & 140 & 11 \\
\hline 380 & HDE 283572 & 041852.5 & +281107 & 6.9 & 8 & wtts & 1,14 & 140 & 11 \\
\hline 404 & V807 Tau & 043005.2 & +240339 & 6.9 & 11 & ctts & 1 & 140 & 11 \\
\hline 76 & UY Aur & 044835.7 & +304214 & 7.0 & 3 & ctts & 1 & 140 & 11 \\
\hline 45 & DK Tau & 042740.5 & +255459 & 7.1 & 9 & ctts & 1 & 140 & 11 \\
\hline 49 & HL Tau & 042844.4 & +180736 & 7.1 & 3 & ctts & 1 & 140 & 11 \\
\hline 50 & XZ Tau & 042846.0 & +180735 & 7.2 & 3 & ctts & 1 & 140 & 11 \\
\hline 415 & HP Tau/G2 & 043254.2 & +224808 & 7.3 & 13 & wtts & 1,14 & 140 & 11 \\
\hline 52 & UZ Tau E & 042939.3 & +254613 & 7.3 & 9 & ctts & 1 & 140 & 11 \\
\hline 374 & Hubble 4 & 041540.9 & +281254 & 7.3 & 11 & wtts & 1 & 140 & 11 \\
\hline 57 & GK Tau & 043032.8 & +241452 & 7.3 & 4 & ctts & 1 & 140 & 11 \\
\hline 66 & HP Tau & 043252.8 & +224818 & 7.3 & 13 & $\mathrm{ctts}$ & 1 & 140 & 11 \\
\hline 402 & FZ Tau & 042930.1 & +241344 & 7.5 & 8 & ctts & 1 & 140 & 11 \\
\hline 386 & FV Tau & 042349.8 & +260013 & 7.5 & 8 & ctts & 1 & 140 & 11 \\
\hline 67 & DO Tau & 043524.2 & +260455 & 7.5 & 7 & ctts & 1 & 140 & 11 \\
\hline 54 & GG Tau & 042937.1 & +172522 & 7.5 & 13 & ctts & 1 & 140 & 11 \\
\hline 29 & V410 Tau & 041524.8 & +282002 & 7.5 & 4 & wtts & 1 & 140 & 11 \\
\hline 396 & Haro 6-13 & 042913.6 & +242243 & 7.6 & 8 & ctts & 1 & 140 & 11 \\
\hline 368 & LkCa 3 & 041142.8 & +274505 & 7.6 & 12 & wtts & 12 & 140 & 11 \\
\hline 56 & GI Tau & 043032.3 & +241503 & 7.6 & 4 & ctts & 1 & 140 & 11 \\
\hline 33 & DE Tau & 041849.8 & +274805 & 7.7 & 7 & ctts & 1 & 140 & 11 \\
\hline 55 & GH Tau & 043004.8 & +240318 & 7.7 & 3 & $\mathrm{ctts}$ & 1 & 140 & 11 \\
\hline 61 & CI Tau & 043052.2 & +224417 & 7.8 & 7 & $\mathrm{ctts}$ & 1 & 140 & 11 \\
\hline 423 & $\mathrm{LkH} \alpha 332 / \mathrm{G} 1$ & 043903.4 & +251724 & 7.8 & 8 & wtts & 8 & 140 & 11 \\
\hline 59 & IS Tau & 043032.7 & +261503 & 8.0 & 4 & ctts & 1 & 140 & 11 \\
\hline 30 & DD Tau & 041525.1 & +280915 & 8.0 & 3 & ctts & 1 & 140 & 11 \\
\hline 41 & IQ Tau & 042647.7 & +26.0016 & 8.0 & 3 & wtts & 1,14 & 140 & 11 \\
\hline 32 & BP Tau & 041608.6 & +285915 & 8.0 & 7 & ctts & 1 & 140 & 11 \\
\hline 398 & V928 Tau & 042917.2 & +241608 & 8.0 & 8 & wtts & 1 & 140 & 11 \\
\hline 44 & FX Tau & 042727.9 & +242018 & 8.1 & 3 & ctts & 1 & 140 & 11 \\
\hline 369 & FO Tau & 041143.6 & +280502 & 8.1 & 8 & ctts & 1 & 140 & 11 \\
\hline 53 & UZ Tau W & 042939.3 & +254613 & 8.1 & 9 & ctts & 1 & 140 & 11 \\
\hline 24 & FN Tau & 041108.6 & +282027 & 8.2 & 3 & ctts & 1 & 140 & 11 \\
\hline 39 & DI Tau & 042638.0 & +262620 & 8.4 & 4 & wtts & 1 & 140 & 11 \\
\hline 388 & $042417+1744$ & 042417.2 & +174403 & 8.4 & 8 & wtts & 1 & 140 & 11 \\
\hline 28 & CY Tau & 041427.7 & +281329 & 8.4 & 3 & ctts & 1 & 140 & 11 \\
\hline 378 & V819 Tau & 041619.9 & +281903 & 8.5 & 8 & wtts & 1 & 140 & 11 \\
\hline
\end{tabular}
(1) Herbig \& Bell (1988)
(2) Rydgren et al. (1976)
(3) Rydgren \& Vrba (1983)
(4) Rydgren \& Vrba (1981)
(5) Warner et al. (1977)

(6) Skrutskie (1992)

(7) Rydgren et al. (1982)

(8) Walter et al. (1988)

(9) Cohen \& Kuhi (1979)

(10) Simon et al. (1992)
(11) Elias (1978)

(12) Herbig et al. (1986)

(13) Moneti \& Zinnecker (1991)

(14) Strom et al. (1989)

\section{DATA ANALYSIS AND RESULTS}

The data obtained for each of the 69 target stars were analyzed using speckle imaging techniques described by Ghez (1992). Both deconvolution of the ensemble averaged autocorrelation function (ACF) of the target star with that of its calibrator and division of their power spectra offered estimates of the object's Fourier amplitudes. Although in theory these methods are Fourier equivalents, in practice they differ (see Sec. 4.1). The Fourier phases for each target were retrieved by calculating the bispectrum of the object and fitting the bispectrum phases using a global least-squares algorithm. A Fourier inversion of these amplitudes and phases was used to produce a final image (see Fig. 1), although the image was unnecessary for the following analysis.

\subsection{Finding Binary Stars and Fitting for their Parameters}

Speckle imaging with the $5 \mathrm{~m}$ telescope spatially resolved much closer binary systems than previously possible with direct imaging by a factor $\sim 20$. Companion stars in the speckle data were initially identified through the CLEAN process of deconvolving the measured object's 
ACF with that of its calibrator (see Gorham et al. 1990). Secondary sources at least $5 \sigma$ above the background noise level are classified as companion stars.

Although calibrated ACFs reveal companion stars, calibrated power spectra were used to provide estimates of their flux density ratio $(R)$ and separation (s). The ACF and power spectra are interchangeable, but a systematic readout noise of the detector is most easily identified and eliminated in the power spectrum domain. In both cases, there is a $180^{\circ}$ ambiguity in the position angle of the binary systems; this is resolved by inspecting the Fourier phases (or equivalently the resulting image).

Figure 1(a), the calibrated and normalized power spectrum for DD Tau, illustrates the characteristic fringe pattern of a binary star,

$$
P(\mathrm{f})=\frac{R^{2}+1+2 R \cos (2 \pi \mathrm{f} \cdot \mathrm{s})}{R^{2}+1+2 R} .
$$

For brighter targets, the object's power spectrum dominates (see Ghez et al. 1991), but spikes typical of the detector readout noise are clearly visible. The data analysis removed these corrupted areas of the power spectrum by setting minimum and maximum allowed values. A template mask excluded other spatial frequencies that (1) contained aliased power due to the slightly oversized pixels (53 as opposed to $45 \mathrm{mas} / \mathrm{pixel}$ ), (2) were outside the diffraction limit, or (3) were less than 2 cycles/arcsec, in order to eliminate the most severe effects of seeing miscalibration (Christou et al. 1985). A one-dimensional power spectrum along the separation axis of the two stars was then generated by averaging the two-dimensional power

TABLE 2(a). Journal of observations Oph-Sco.

\begin{tabular}{clccc}
\hline \hline Date & \multicolumn{1}{c}{ Object } & Cal (SAO) & Sets & Seeing at K (") \\
\hline 1990 July 7 & AS 205 & 159745 & 3 & 0.9 \\
& RNO 90 & 159948 & 4 & 0.7 \\
1990 July 8 & 155913-2233 & 183901 & 5 & 0.8 \\
& 155203-2338 & 183901 & 4 & 0.7 \\
& 162218-2420 & 183901 & 4 & 0.7 \\
& ROXs 42C & 184549 & 5 & 0.8 \\
& ROXs 43A & 184549 & 4 & 0.7 \\
1990 July 9 & DoAr 21 & 184429 & 4 & 0.9 \\
& SR 24 S & 184549 & 4 & 0.9 \\
& DoAr 24 E & 184549 & 6 & 0.9 \\
& SR 9 & 184549 & 4 & 0.9 \\
& SR 20 & 184549 & 4 & 0.9 \\
& Haro 1-16 & 184549 & 4 & 1.2 \\
& Haro 1-4 & 184549 & 4 & 0.9 \\
1992 Feb 18 & Haro 1-14 & 184429 & 6 & 1.0 \\
\hline \hline & SR 4 & 184549 & 4 & 0.9 \\
1990 Aug 5 Aug 6 & SR 9 & 184549 & 5 & 0.9 \\
& 160946-1851 & 159745 & 4 & 0.9 \\
& 160815-1857 & 159745 & 4 & 0.9 \\
& Haro 1-16 & 184549 & 4 & 0.9 \\
& 160827-1813 & 159745 & 6 & 1.0 \\
& SR 4 & 184549 & 8 & 1.0 \\
& V853 Oph & 184549 & 8 & 0.8 \\
& DoAr 24 & 184549 & 6 & 1.0 \\
& 155828-2232 & 183901 & 4 & 1.4 \\
& ROXs 42C & 184549 & 6 & 1.2 \\
& & & & 1.0 \\
\hline
\end{tabular}

TABLE 2(b). Journal of observations Tau-Aur.

\begin{tabular}{|c|c|c|c|c|}
\hline Date & Object & Cal (SAO) & Sets & Seeing at $\mathrm{K}(")$ \\
\hline \multirow[t]{3}{*}{1990 Oct 2} & RY Tau & 76461 & 8 & 1.0 \\
\hline & DD Tau & 76551 & 8 & 1.1 \\
\hline & $\mathrm{LkH} \alpha 332 / \mathrm{G} 1$ & 76727 & 4 & 1.2 \\
\hline 1990 Oct 3 & V773 Tau & 76551 & 8 & 1.0 \\
\hline \multirow[t]{5}{*}{1990 Oct 4} & CW Tau & 76551 & 8 & 0.8 \\
\hline & DD Tau & 76551 & 8 & 0.7 \\
\hline & RY Tau & 76461 & 8 & 0.7 \\
\hline & $\mathrm{LkH} \alpha 332 / \mathrm{G} 1$ & 76727 & 8 & 0.7 \\
\hline & SU Aur & 57454 & 10 & 0.6 \\
\hline \multirow[t]{4}{*}{1990 Nov 9} & DG Tau & 76613 & 9 & 1.3 \\
\hline & DR Tau & 93907 & 8 & 1.3 \\
\hline & RW Aur & 76876 & 8 & 0.9 \\
\hline & DF Tau & 76613 & 5 & 1.0 \\
\hline \multirow[t]{6}{*}{1990 Nov 10} & HDE 283572 & 76551 & 3 & 1.2 \\
\hline & V807 Tau & 76613 & 8 & 0.9 \\
\hline & UZ Tau E & 76613 & 8 & 0.9 \\
\hline & UZ Tau W & 76613 & 8 & 0.9 \\
\hline & UY Aur & 57454 & 8 & 1.2 \\
\hline & HL Tau & 93907 & 11 & 1.2 \\
\hline \multirow[t]{7}{*}{1991 Oct 18} & LkCa 3 & 76461 & 2 & 0.6 \\
\hline & FO Tau & 76461 & 5 & 0.6 \\
\hline & CY Tau & 76461 & 4 & 0.6 \\
\hline & V819 Tau & 76461 & 6 & 0.6 \\
\hline & DI Tau & 76727 & 4 & 0.6 \\
\hline & FX Tau & 76727 & 2 & 0.6 \\
\hline & $042417+1744$ & 93918 & 5 & 0.6 \\
\hline \multirow[t]{10}{*}{1991 Oct 19} & FN Tau & 76461 & 6 & 0.5 \\
\hline & BP Tau & 76461 & 6 & 0.5 \\
\hline & IQ Tau & 76727 & 4 & 0.5 \\
\hline & V928 Tau & 76727 & 6 & 0.6 \\
\hline & IS Tau & 76727 & 6 & 0.6 \\
\hline & FZ Tau & 76727 & 6 & 0.6 \\
\hline & Haro 6-13 & 76727 & 6 & 0.5 \\
\hline & GI Tau & 76727 & 6 & 0.6 \\
\hline & GH Tau & 76727 & 6 & 0.6 \\
\hline & CI Tau & 76727 & 4 & 0.5 \\
\hline \multirow[t]{10}{*}{1991 Oct 20} & V410 Tau & 76461 & 6 & 0.7 \\
\hline & DE Tau & 76485 & 7 & 1.1 \\
\hline & Hubble 4 & 76485 & 6 & 0.8 \\
\hline & GK Tau & 76727 & 5 & 0.8 \\
\hline & FV Tau & 76613 & 6 & 0.7 \\
\hline & GG Tau & 93918 & 6 & 0.7 \\
\hline & HP Tau & 76727 & 5 & 0.8 \\
\hline & HP Tau/G2 & 76727 & 5 & 0.8 \\
\hline & DK Tau & 76727 & 5 & 0.8 \\
\hline & XZ Tau & 93874 & 5 & 1.0 \\
\hline 1991 Nov 18 & T Tau & 98918 & 4 & 1.4 \\
\hline 1991 Nov 19 & DO Tau & 76618 & 5 & 0.9 \\
\hline
\end{tabular}

spectrum in the direction perpendicular to the separation axis. Fits to this one-dimensional power spectrum provided estimates of the binary system flux density ratio and separation. Figure 2 shows the one-dimensional power spectrum for DD Tau and the corresponding model fit.

Two stars warrant special attention: in the widely separated $(s>2$ ") binary, DK Tau, the companion star is detected, but much of its flux fell off the edge of the field of view. The binary star parameters were not well determined from these observations and are not reported here.

Figure 3 shows components of the second case, the triple system RW Tau, labeled A, B, and C in order of decreasing $2.2 \mu \mathrm{m}$ flux density. The analysis of this system proceeded in two steps, each of which dealt with the inter- 

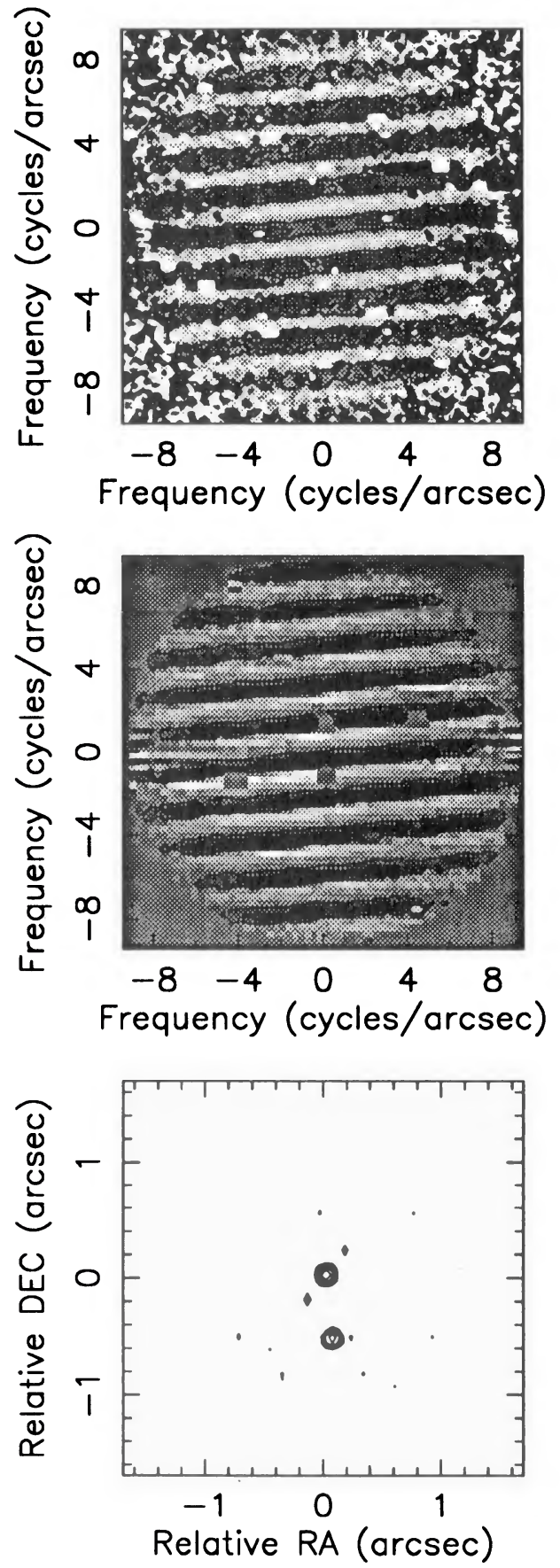

FIG. 1. The reconstruction of the binary star DD Tau from 1990 Oct 4 (a) the calibrated power spectrum (b) the Fourier phases, and (c) the final image.

ference effects between a set of components. A fit to the lower spatial frequencies of the power spectrum projected along the A-B separation axis provided estimates of the parameters of the wide binary system. This is equivalent to an observation with poorer resolution, which could not resolve component $\mathrm{C}$. The parameters obtained in this first step are for A-(BC) (i.e., the flux density ratio is between star A and the combined light of B and C). Likewise a fit to the power spectrum projection along the $\mathrm{B}-\mathrm{C}$ separation axis determined the parameters of this close pair.

Among the 69 target objects observed, one triple and 31

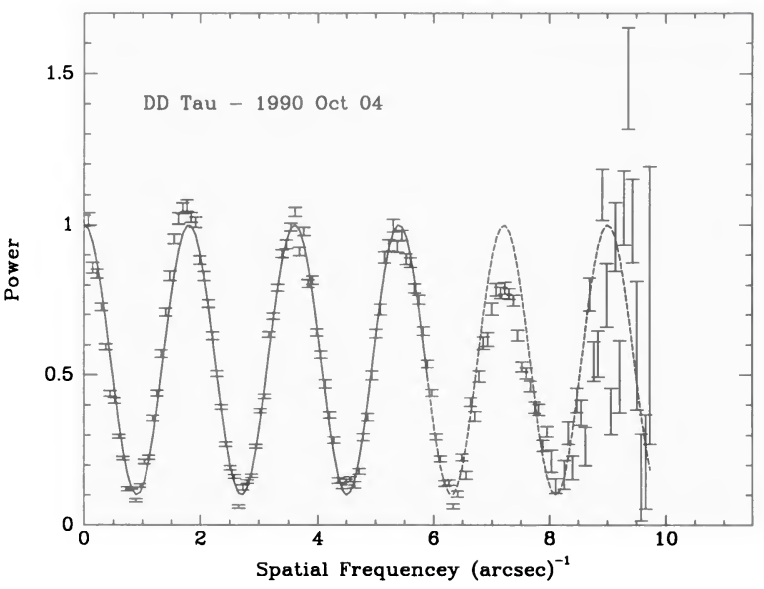

FIG. 2. The one-dimensional power spectrum and model fit for DD Tau. The solid line is over the points that were included in the fit and the dashed line is the extension of the resulting model fit.

binary systems were detected; 9 are new detections. Table 3 lists the fitted binary system parameters and their uncertainties. The relative $K$ band flux of the stars at the time of the observation set their designation as primary, secondary, or tertiary (A, B, and C), where the brightest component is taken as the primary star; the available data did not permit mass estimates for the companion stars. $T$ Tauri stars are known to be variable, so relative brightnesses may well change with time.

The probability that any of the detected double stars are merely chance alignments with a field star is small. Simon et al. (1992) estimate that the density of field stars with $K$ magnitudes brighter than $12 \mathrm{mag}$ is $\approx 4 \times 10^{-5}$ stars/arcsec ${ }^{2}$ in the direction of Tau-Aur and $\approx 3 \times 10^{-4}$ stars/ $\operatorname{arcsec}^{2}$ in the direction of Oph-Sco (Simon 1992b).

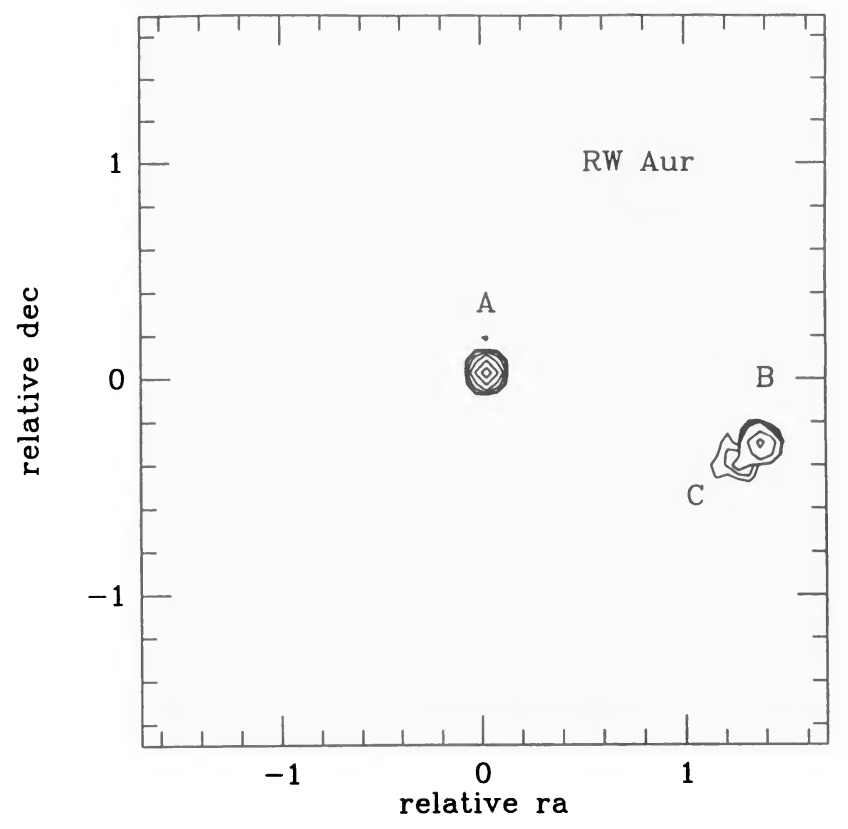

FIG. 3. The speckle image of RW Aur shows a newly discovered third component. 
The faintest companion observed has a $K=11 \mathrm{mag}$ (Fig. 7). As discussed in Sec. 4.2 below, the largest measured binary star separation is $\sim 2$."5. Of the 45 targets in TauAur, 0.04 are therefore expected to show a field star within a radius of 2".5. Likewise, for the 24 targets observed in Oph-Sco, only 0.14 are expected to appear as doubles as a result of a chance projection with a field star.

\subsection{Limits for Undetected Companion Stars}

The sensitivity of a given observation depends on several factors: (1) the total flux density of the target, (2) the "seeing," or the coherence length $\left(r_{0}\right)$ of the atmosphere, and its stability, (3) the atmospheric coherence time $\left(\tau_{0}\right)$ and its stability, (4) the amount of data accumulated on the target, and (5) the systematic electronic readout noise (the strength of which varied from run to run). Each factor has a different effect on the way the sensitivity varies as a function of separation and most of these factors varied from observation to observation. Thus the sensitivity of each observation to finding a companion star as a function of separation is unique. It is essential to evaluate these effects to estimate the completeness of the survey.

In theory, the angular separation range of binary stars detectable by this survey should be constrained only by the diffraction limit of the Hale $5 \mathrm{~m}$ Telescope and the field of view of the camera. At $\lambda=2.2 \mu \mathrm{m}$, the diffraction limit corresponds to a maximum spatial frequency, $f_{\max }$, of 11.2 cycles/arcsec. As described earlier the existence of a binary system is revealed in the power spectrum by a sinusoid. Thus the limiting binary star angular separation, 0.045 , is set by a binary star whose first minimum occurs at $f_{\max }$.
The upper limit for the survey's binary star separation range depends on the camera's field of view. Each target star observed was centered on the array and thus any components outside half the field of view (plus half the seeing disk) were not detected. Since the detector is not circular, the maximum binary star separation that could have been observed depends on the position angle and ranges from 1 ." 8 to 2.5 , where the effects of the array size and the best seeing conditions of 0.5 (see Table 2) are considered to give a conservative upper limit. An upper limit of 1".8 for the angular separation range eliminates the dependence on position angle.

Within the range of separation of 0.045 to 1 ". 8 , the sensitivity to faint companions is not uniform and increases with increasing separation. In particular, the sensitivity tends to roll off between 0.1 and 0.045 because the exposure time used $(100 \mathrm{~ms})$ did not always match the atmospheric conditions. For example, when the atmospheric coherence time was shorter than the exposure time, the highest spatial frequencies were suppressed.

Limits for undetected companion stars at a number of separations can be determined empirically for each observation from the noise level in the power spectrum. The algorithm used was originally suggested by Henry (1991) and is based on the minimum values observed in a group of one-dimensional power spectra made from projections of $P(\mathbf{f})$ at different assumed position angles of an undetected binary star. For each target, 36 one-dimensional power spectra, $p(f)$, are produced by projecting the twodimensional power spectrum, $P(\mathbf{f})$, at angles between $0^{\circ}$ and $175^{\circ}$, with respect to the sky, at $5^{\circ}$ intervals. The power spectrum is symmetric and therefore the projections be-

TABle 3(a). Double star parameters Oph-Sco.

\begin{tabular}{|c|c|c|c|c|c|}
\hline Object & Date & $\begin{array}{l}\text { Separation } \\
\text { (arcsec) }\end{array}$ & $\begin{array}{l}\text { P.A. } \\
\text { (deg) }\end{array}$ & Flux Ratio & Notes \\
\hline SR 20 & 1990 July 9 & $0.071 \pm 0.001$ & $225 \pm 5$ & $8 \pm 1$ & \\
\hline \multirow[t]{2}{*}{ ROXs $42 \mathrm{C}$} & 1990 July 8 & $0.157 \pm 0.003$ & $135 \pm 3$ & $4.0 \pm 0.34$ & $5 a$ \\
\hline & 1991 May 3 & $0.152 \pm 0.003$ & $139 \pm 3$ & $4.7 \pm 0.3$ & \\
\hline $160946-1851$ & 1990 Aug 5 & $0.208 \pm 0.004$ & $164 \pm 2$ & $3.99 \pm 0.06$ & \\
\hline $162218-2420$ & 1990 July 8 & $0.236 \pm 0.005$ & $156 \pm 2$ & $1.25 \pm 0.03$ & 4 \\
\hline $155913-2233$ & 1990 July 8 & $0.288 \pm 0.005$ & $347 \pm 2$ & $2.15 \pm 0.03$ & $5 a$ \\
\hline V853 Oph & 1990 Aug 7 & $0.399 \pm 0.008$ & $96 \pm 2$ & $4.2 \pm 0.5$ & \\
\hline \multirow[t]{2}{*}{ SR 9} & 1990 July 9 & $0.59 \pm 0.01$ & $350 \pm 1$ & $11 \pm 2$ & 2 \\
\hline & 1990 Aug 5 & $0.59 \pm 0.01$ & $350 \pm 1$ & $13 \pm 2$ & \\
\hline Haro 1-4 & 1990 July 9 & $0.72 \pm 0.01$ & $27 \pm 1$ & $4.2 \pm 0.2$ & 2 \\
\hline $155203-2338$ & 1990 July 8 & $0.80 \pm 0.01$ & $229 \pm 1$ & $7.1 \pm 0.4$ & \\
\hline AS 205 & 1990 July 7 & $1.32 \pm 0.02$ & $212 \pm 1$ & $3.2 \pm 0.2$ & 2,3 \\
\hline DoAr $24 \mathrm{E}$ & 1990 July 9 & $2.03 \pm 0.04$ & $150 \pm 1$ & $5.6 \pm 0.9$ & 1 \\
\hline
\end{tabular}

Notes proceeded by an "a" indicate measurements of additional components in these systems that were outside of the separation range of this survey. Otherwise the notes refer to other measurements of the observed binary stars.

(1) Chelli et al. (1988) identified the companion using direct near infrared imaging techniques

(2) Weintraub (1989) detected these binary stars using one-dimensional near-infrared speckle measurements. The discrepancies between these results and the survey presented here are easily accounted for by the limited angular resolution obtained by Weintraub (1989) $\left(f_{\max }=0.3-3.6\right.$ cycles/arcsec $)$

(3) Cohen \& Kuhi (1979) identified the secondary component directly at optical wavelengths

(4) Aitken (1939) and Heinz (1980) detected this companion at optical wavelengths with micrometer measurements.

(5a) Mathieu et al. (1988) detected both a spectroscopic binary companion in both 162814-2427 (ROXs 42C)and 155913-2233 thus both systems consist of at least three stars. 
TABLE 3(b). Double star parameters Tau-Aur.

\begin{tabular}{|c|c|c|c|c|c|}
\hline Object & Date & $\begin{array}{c}\text { Separation } \\
\text { (arcsec) }\end{array}$ & $\begin{array}{l}\text { P.A. } \\
\text { (deg) }\end{array}$ & Flux Ratio & Notes \\
\hline DF Tau & 1990 Nov 9 & $0.088 \pm 0.002$ & $329 \pm 5$ & $2.13 \pm 0.02$ & 1 \\
\hline V773 Tau & 1990 Oct 3 & $0.112 \pm 0.002$ & $295 \pm 4$ & $2.15 \pm 0.01$ & 8 \\
\hline DI Tau & 1991 Oct 18 & $0.12 \pm 0.01$ & $294 \pm 4$ & $8 \pm 1$ & 1 \\
\hline V410 Tau & 1991 Oct 20 & $0.123 \pm 0.002$ & $218 \pm 4$ & $6.2 \pm 0.9$ & \\
\hline FO Tau & 1991 Oct 18 & $0.166 \pm 0.003$ & $182 \pm 3$ & $1.53 \pm 0.03$ & 8 \\
\hline V928 Tau & 1991 Oct 19 & $0.165 \pm 0.003$ & $125 \pm 3$ & $1.66 \pm 0.02$ & 8 \\
\hline \multirow[t]{2}{*}{$\mathrm{LkH} \alpha 332 / \mathrm{G} 1$} & 1990 Oct 4 & $0.215 \pm 0.004$ & $77 \pm 2$ & $1.78 \pm 0.02$ & 8 \\
\hline & 1990 Oct 2 & $0.201 \pm 0.004$ & $261 \pm 2$ & $3.4 \pm 0.2$ & \\
\hline IS Tau & 1991 Oct 19 & $0.221 \pm 0.004$ & $92 \pm 2$ & $6.3 \pm 0.9$ & 1 \\
\hline GG Tau & 1991 Oct 20 & $0.288 \pm 0.006$ & $3 \pm 2$ & $4.0 \pm 0.1$ & $6,10 \mathrm{a}$ \\
\hline $\mathrm{XZ}$ Tau & 1991 Oct 20 & $0.311 \pm 0.006$ & $153 \pm 2$ & $1.96 \pm 0.05$ & 7 \\
\hline GH Tau & 1991 Oct 19 & $0.314 \pm 0.006$ & $299 \pm 2$ & $1.8 \pm 0.02$ & 8 \\
\hline UZ Tau W & 1990 Nov 10 & $0.360 \pm 0.007$ & $359 \pm 1$ & $2.18 \pm 0.07$ & 1 \\
\hline V807 Tau & 1990 Nov 9 & $0.375 \pm 0.007$ & $330 \pm 1$ & $2.69 \pm 0.06$ & $11 \mathrm{a}, 8$ \\
\hline LkCa 3 & 1991 Oct 18 & $0.491 \pm 0.009$ & $77 \pm 1$ & $1.046 \pm 0.005$ & 8 \\
\hline \multirow[t]{2}{*}{ DD Tau } & 1990 Oct 4 & $0.56 \pm 0.01$ & $186 \pm 1$ & $1.92 \pm 0.02$ & \\
\hline & 1990 Oct 2 & $0.56 \pm 0.01$ & $186 \pm 1$ & $2.17 \pm 0.07$ & 3,8 \\
\hline T Tau. & 1991 Nov 18 & $0.71 \pm 0.01$ & $176 \pm 1$ & $11 \pm 1$ & 4 \\
\hline FV Tau & 1991 Oct 20 & $0.73 \pm 0.01$ & $92 \pm 1$ & $1.56 \pm 0.04$ & $1,6,9 a$ \\
\hline UY Aur & 1990 Nov 10 & $0.88 \pm 0.02$ & $227 \pm 1$ & $2.86 \pm 0.06$ & $5,2,8$ \\
\hline FX Tau & 1991 Oct 18 & $0.90 \pm 0.02$ & $291 \pm 1$ & $1.965 \pm 0.001$ & \\
\hline RW Aur A-BC & 1990 Nov 9 & $1.39 \pm 0.03$ & $256 \pm 1$ & $8 \pm 1$ & $5,2,8$ \\
\hline RW Aur B-C & & $0.120 \pm 0.004$ & $111 \pm 3$ & $41 \pm 105$ & \\
\hline DK Tau & 1991 Oct 20 & - & - & - & 1,2 \\
\hline
\end{tabular}

Notes proceeded by an "a" indicate measurements of additional components in these systems that were outside of the separation range of this survey. Otherwise the notes refer to other measurements of the observed binary stars.

(1) Simon et al.'s (1992) measured the binary systems DF, DI, FV, and UZ Tau E using lunar occultations and DK Tau using direct imaging techniques. Note: Although IS Tau was initially reported as a single star, the companion was detected in the occultation measurements close to the noise level (Simon 1992b).

(2) Weintraub (1989) detected these binary stars using one-dimensional near-infrared speckle measurements. The discrepancies between these results and the survey presented here are easily accounted for by the limited angular resolution obtained by Weintraub (1989) $\left(f_{\max }=0.3-3.6\right.$ cycles/arcsec $)$

(3) Bouvier et al. (1992) studied this double star at multiple wavelengths using speckle and direct imaging techniques

(4) This double star was first detected by Dyck et al. (1982) with one-dimensional near-infrared speckle interferometry. Ghez et al. (1991) and references within have also studied this double star using high resolution imaging techniques.

(5) Joy \& Van Biesbroeck (1944) resolved the widely separated components of RW Aur and UY Aur directly at optical wavelengths.

(6) Leinert et al. (1991) measured the double star GG Tau using one-dimensional speckle imaging techniques and FV Tau using lunar occultations. speckle, and CCD imaging

(7) Haas et al. (1990) measured this double star using one-dimensional near-infrared speckle imaging techniques

(8) Leinert et al. (1992) measure these double stars using near infrared speckle imaging techniques.

(9a) FV Tau appears to consist of at least four stars. FV Tau/c is located 12" away from FV Tau (Herbig \& Bell 1988). Both FV Tau (see table) and FV Tau/c have close companions (Simon et al. 1992).

(10a) GG Tau also appears to consist of at least four stars. GG Tau/c is located 10" away from GG Tau (Cohen \& Kuhi 1979, Moneti \& Zinnecker 1991). Both GG Tau (see table) and GG Tau/c have close companions detected by Leinert et al. (1991).

(11a) Simon (1992b) detected an additional companion with a projected separation of 23 mas from the southern component of the observed binary star V807 Tau (Elias 12). This third component reduces the visibility in our measurements, but no minimum is observed, so the actual separation is between $23-45$ mas.

tween $180^{\circ}$ and $355^{\circ}$ offer no new information. As described in Sec. 4.1, certain regions are excluded from these projections.

The minimum values found in the projected power spectra characterize the limit of the flux density ratio (primary/secondary $\geqslant 1$ ) of an undetected binary system. The two-dimensional power spectrum of an unresolved source is completely flat in all directions, $P(\mathbf{f})=1$. For a binary star, $P(f)$ varies sinusoidally along an axis parallel to the binary star separation vector. The amplitude depends on the flux density ratio of the system and the period depends on the magnitude of the binary star separation. The one-dimensional power spectra, $p(f)$, obtained from projections at angles near the separation vector of the binary star should reveal this variation. In contrast, at angles perpendicular to the binary axis, $p(f)$ should be close to unity at all spatial frequencies. The power spectra at the spatial frequency where a first minimum is expected for a 
given binary star separation $\left[f_{1 \text { st } \min }=1 /(2 s)\right]$, sets an upper limit to the flux density ratio for an undetected companion. Minima of the normalized power spectrum translate into flux density ratio limits by the following relationship

$$
\text { flux ratio limit }=\frac{1+\sqrt{\text { minimum power }}}{1-\sqrt{\text { minimum power }}} .
$$

Since the noise in the power spectrum increases with spatial frequency, limits at many different binary star separations were calculated; Table 4 lists those at $0.1,0.2$, and 0 ."8. Each projection is normalized by the mean of the projected values at zero frequency $[p(0)]$ and the variance of these levels determines the uncertainty quoted for each limit. Figure 4 shows examples of the projected power spectra of sources for which no binary component was seen.

Limits for additional companions can also be obtained for those observations in which multiple systems are detected. The evidence of the observed companions is first removed by expanding the one-dimensional binary star solution to a two-dimensional function and subtracting it from the original data. The residuals are then treated as described above for the single star case. Table 5 contains these limits for additional components in the multiple star systems.

The average limiting flux density ratio for undetected companion stars among the 69 observed targets provides an estimate of the overall survey sensitivity (as opposed to that of the individual observations) for finding faint companion stars. In cases of multiple observations of the same object only the best measurement is included in the average. Figure 5 shows histograms of the limiting flux density ratios at separations of $0.045,0.055,0.069,0$."08, 0. $1,0.2$, and 0.8 , with the mean and the rms value printed in the top right corner of each distribution. The bin farthest to the left in each of the histograms contains observations that have no significant signal at that particular angular resolution, i.e., a $\Delta K=0$ mag binary star would not have been detected.

Figure 6 shows the average limiting sensitivity curve for this survey constructed from the mean values of the distributions in Fig. 5. The circles in Fig. 6 indicate the observed magnitude differences of the detected binary stars. On average, the observations are of sufficient quality to detect binary stars with magnitude differences of up to $3.1 \mathrm{mag}$ for separations between 0.1 and 1.8. Speckle images of individual objects are routinely made with a much higher dynamic range (e.g., Ghez et al. 1991; Koresko et al. 1991; Henry et al. 1992) by obtaining larger data sets. However, a lower overall sensitivity was accepted here in the interest of observing many objects.

Figure 7 shows a different average sensitivity curve evaluated in terms of the limiting $K$ magnitudes for additional companion stars. Its construction follows that of the one shown in Fig. 6 but the flux density ratio limits are combined with the $K$ magnitude of each target found in the literature (Table 1). On average, the observations are sensitive to companion stars brighter than $K=10.6 \mathrm{mag}$ at distances between 0.1 and 1". 8 from the target stars. This is consistent with the estimated apparent magnitudes of the detected companion stars, which are also plotted in Fig. 7.

\section{DISCUSSION \\ 5.1 Completeness and Multiplicity}

The first few sections of this discussion (Secs. 5.2-5.4) compare the binary star frequencies of various groups of $\mathrm{T}$ Tauri stars. To ensure consistency in these comparisons, a subset of the survey is defined as the "complete sample." The first step taken to construct this sample requires each of the included observations, whether or not a companion star was detected, to be sensitive to additional companions within a specified region of the magnitude differenceangular separation phase space. This region, the "completeness region," has been defined to guarantee the detection of all the companion stars with magnitude difference and angular separation within the completeness region for sources in the complex sample. Based on the average sensitivity curve plotted in Fig. 6, the completeness region is restricted to angular separations between 0 ." 1 and 1.8 and magnitude differences between 0.0 and $2.0 \mathrm{mag}$. Insufficient sensitivity excludes five of the 69 targets observed (155828-2232, 160905-1859, DE Tau, 155913-2233, and DK Tau from the complete sample. Two of the excluded sources are detected as double stars. A second step taken to construct the complete sample converts the angular separation range to a projected linear separation range, since the assumed distances to the star forming regions studied range from 140 to $160 \mathrm{pc}$ (see Column 9 in Table 1). The angular separation range of 0 ". 1 to 1.8 corresponds to projected linear separations of 16 to $288 \mathrm{AU}$ and 14 to $252 \mathrm{AU}$ at the maximum distance of $160 \mathrm{pc}$ and the minimum distance of $140 \mathrm{pc}$, respectively. Thus the complete sample incorporates only the common range of 16 to $252 \mathrm{AU}$.

Figure 8 shows the distribution of detected companions for targets in the complete sample. Twenty-two companion stars are included within the completeness region. Outside the boundaries of the completeness region (the outlined area in Fig. 8), the detection of companion stars, such as those of RW Aur, T Tau, and SR 9, is no longer guaranteed. Some observations do show companions outside this region because they have better sensitivity than is required to be a member of the complete sample.

In discussing the data, it is useful to define the true binary star frequency, the ratio of the number of companion stars with any separation and magnitude difference to the number of independent systems a given sample:

$$
\mathrm{BSF}=\int_{0}^{\infty} \int_{0}^{\infty} \frac{d \mathrm{BSF}}{d s d \Delta K} d s d \Delta K .
$$

In a specific observational program, the restricted binary star frequency, the bsf, defined as the ratio of companion stars to the number of targets in the completeness region, is measured rather than the BSF. This restricted binary star frequency can be expressed in terms of a differential binary star frequency, $d \mathrm{BSF} / d s d \Delta K$, 
TABLE 4(a). Limits for stars observed without companion stars in Oph-Sco.

\begin{tabular}{|c|c|c|c|c|c|}
\hline \multirow[t]{2}{*}{ Object } & \multirow[t]{2}{*}{ Date } & \multicolumn{3}{|c|}{ Flux Ratio Limits } & \multirow[t]{2}{*}{ Notes } \\
\hline & & $0 . " 10$ & $0 . " 20$ & $0 . " 80$ & \\
\hline V1121 Oph & 1990 July 7 & $47 \pm 13$ & $47 \pm 13$ & $47 \pm 13$ & \\
\hline RNO 90 & 1990 July 7 & $27 \pm 7$ & $27 \pm 7$ & $28 \pm 8$ & \\
\hline SR $24 \mathrm{~S}$ & 1990 July 9 & $23 \pm 7$ & $23 \pm 7$ & $23 \pm 7$ & $1 \mathrm{a}$ \\
\hline \multirow[t]{2}{*}{ Haro 1-16 } & 1990 Aug 6 & $20 \pm 4$ & $20 \pm 4$ & $20 \pm 4$ & \\
\hline & 1990 July 9 & $14 \pm 3$ & $14 \pm 3$ & $14 \pm 3$ & \\
\hline $160815-1857$ & 1990 Aug 5 & $17 \pm 3$ & $18 \pm 4$ & $18 \pm 4$ & $3 \mathbf{a}$ \\
\hline \multirow[t]{2}{*}{ SR 4} & 1990 Aug 7 & $17 \pm 4$ & $22 \pm 6$ & $23 \pm 6$ & \\
\hline & 1990 July 9 & $11 \pm 2$ & $11 \pm 2$ & $11 \pm 2$ & \\
\hline DoAr 21 & 1990 July 9 & $16 \pm 2$ & $16 \pm 2$ & $16 \pm 2$ & \\
\hline ROXs 43A & 1990 July 8 & $14 \pm 4$ & $14 \pm 4$ & $14 \pm 4$ & $2 a$ \\
\hline $160827-1813$ & 1990 Aug 6 & $11 \pm 2$ & $11 \pm 2$ & $11 \pm 2$ & \\
\hline DoAr 24 & 1990 Aug 7 & $8.0 \pm 0.8$ & $15 \pm 3$ & $15 \pm 3$ & \\
\hline Haro 1-14 & 1992 Feb 18 & $8 \pm 2$ & $8 \pm 2$ & $10 \pm 3$ & \\
\hline $160905-1859$ & 1990 Aug 5 & $3.2 \pm 0.3$ & $5.1 \pm 0.7$ & $5.4 \pm 0.8$ & \\
\hline $155828-2232$ & 1990 Aug 7 & $3.0 \pm 0.7$ & $3.0 \pm 0.7$ & $3.0 \pm 0.7$ & \\
\hline
\end{tabular}

Notes proceeded by an "a" indicate measurements of additional components in these systems that were outside of the separation range of this survey.

(1a) Haro \& Chavira (1974) noted SR 24 to be a wide (6" at P.A. $60^{\circ}$ ) north - south double star. Simon (1992b) found SR 24 S to be associated with an additional close companion star.

(2a) ROXs 43 appears to consist of at least three stars. Bouvier \& Appenzeller (1991) identified the widely separated (6" at P.A. $13^{\circ}$ ) pair, which they labeled as ROX $43 \mathrm{AB}$. Mathieu et al. (1988) measured 162819-2423S (ROX 43A) as a spectroscopic binary system.

(3a) Mathieu et al. (1988) discovered a spectroscopic binary in this system.

TABLE 4(b). Limits for stars observed without companion stars in Tau-Aur.

\begin{tabular}{|c|c|c|c|c|c|}
\hline \multirow[t]{2}{*}{ Object } & \multirow[t]{2}{*}{ Date } & \multicolumn{3}{|c|}{ Flux Ratio Limits } & \multirow[t]{2}{*}{ Notes } \\
\hline & & $0 . " 10$ & $0 . " 20$ & $0 . " 80$ & \\
\hline$\overline{\text { DR Tau }}$ & 1990 Nov 9 & $43 \pm 11$ & $43 \pm 11$ & $43 \pm 11$ & \\
\hline \multirow[t]{2}{*}{ RY Tau } & 1990 Oct 2 & $43 \pm 12$ & $43 \pm 12$ & $45 \pm 13$ & \\
\hline & 1990 Oct 4 & $27 \pm 8$ & $27 \pm 8$ & $27 \pm 8$ & \\
\hline SU Aur & 1990 Oct 4 & $41 \pm 14$ & $44 \pm 16$ & $44 \pm 16$ & \\
\hline CW Tau & 1990 Oct 4 & $28 \pm 7$ & $34 \pm 9$ & $35 \pm 10$ & \\
\hline BP Tau & 1991 Oct 19 & $26 \pm 7$ & $30 \pm 10$ & $35 \pm 12$ & \\
\hline Haro 6-13 & 1991 Oct 19 & $25 \pm 8$ & $25 \pm 8$ & $25 \pm 8$ & \\
\hline FZ Tau & 1991 Oct 19 & $25 \pm 8$ & $25 \pm 8$ & $25 \pm 8$ & \\
\hline DO Tau & 1991 Nov 19 & $24 \pm 6$ & $24 \pm 6$ & $24 \pm 6$ & \\
\hline UZ Tau E & 1990 Nov 10 & $22 \pm 6$ & $22 \pm 6$ & $23 \pm 7$ & \\
\hline IQ Tau & 1991 Oct 19 & $18 \pm 4$ & $18 \pm 4$ & $18 \pm 4$ & $1 \mathrm{a}$ \\
\hline FN Tau & 1991 Oct 19 & $18 \pm 5$ & $18 \pm 5$ & $18 \pm 5$ & \\
\hline HL Tau & 1990 Nov 10 & $16 \pm 4$ & $20 \pm 6$ & $20 \pm 6$ & \\
\hline GI Tau & 1991 Oct 19 & $16 \pm 6$ & $16 \pm 6$ & $16 \pm 6$ & \\
\hline $042417+1744$ & 1991 Oct 18 & $15 \pm 3$ & $16 \pm 3$ & $16 \pm 3$ & \\
\hline DG Tau & 1990 Nov 9 & $14 \pm 4$ & $22 \pm 7$ & $22 \pm 7$ & \\
\hline V819 Tau & 1991 Oct 18 & $14 \pm 4$ & $14 \pm 4$ & $14 \pm 4$ & \\
\hline CY Tau & 1991 Oct 18 & $14 \pm 4$ & $14 \pm 4$ & $14 \pm 4$ & \\
\hline HP Tau & 1991 Oct 20 & $11 \pm 2$ & $15 \pm 4$ & $16 \pm 5$ & \\
\hline GK Tau & 1991 Oct 20 & $11 \pm 4$ & $11 \pm 4$ & $11 \pm 4$ & \\
\hline CI Tau & 1991 Oct 19 & $10 \pm 2$ & $10 \pm 2$ & $10 \pm 2$ & \\
\hline HDE 283572 & 1990 Nov 10 & $9 \pm 2$ & $9 \pm 2$ & $9 \pm 2$ & \\
\hline HP Tau/G2 & 1991 Oct 20 & $9 \pm 3$ & $9 \pm 3$ & $9 \pm 3$ & $2 a$ \\
\hline Hubble 4 & 1991 Oct 20 & $8 \pm 2$ & $8 \pm 2$ & $8 \pm 2$ & \\
\hline DE Tau & 1991 Oct 20 & $6 \pm 1$ & $8 \pm 2$ & $12 \pm 4$ & \\
\hline
\end{tabular}

Notes proceeded by an "a" indicate measurements of additional components in these systems that were outside of the separation range of this survey.

(1a) Herbig \& Bell (1988) detected another star separated by 10" from IQ Tau.

(2a) HP Tau/G2 is located 10" away from HP Tau/G3 (Moneti \& Zinnecker 1991) 

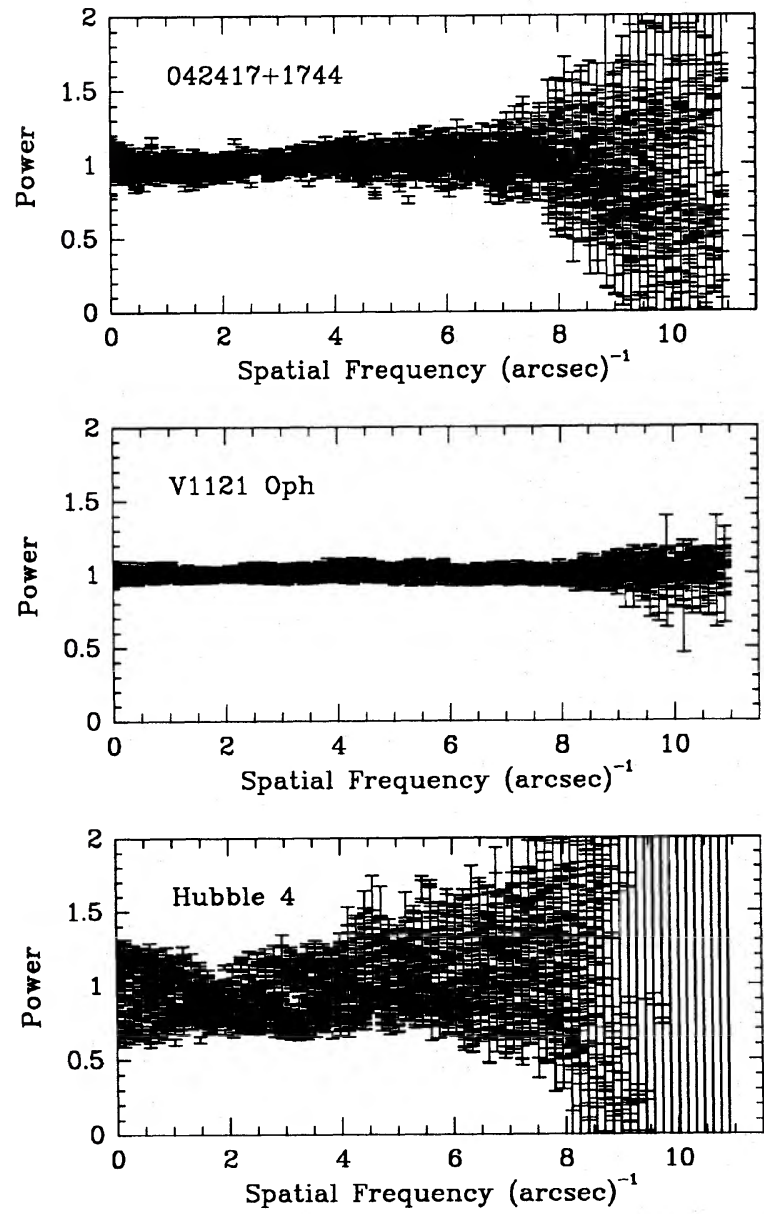

FIG. 4. The 36 one-dimensional power spectra used to place limits on undetected companion stars (see Sec. 4.2) for V1121 Oph, 042417 +1744 , and Hubble 4. These three examples show the range of sensitivity obtained in this survey. V1121 Oph is an example of one of the best limits achieved and Hubble 4 is an example of one of the poorest sensitivity observations allowed in the complete sample (see Sec. 5.1).

$$
\mathrm{bsf}=\int_{0 \mathrm{mag}}^{2 \mathrm{mag}} \int_{16 \mathrm{AU}}^{252 \mathrm{AU}} \frac{d \mathrm{BSF}}{d s d \Delta K} d s d \Delta K,
$$

where $s$ is the projected linear separation, $\Delta K$ is the $K$ magnitude difference, and BSF is the total or "true" binary star frequency. Within the completeness region there are 64 targets and 22 companion stars (see Fig. 8); thus the restricted binary star frequency is $34[ \pm 7] \%$.

The target stars with no companions or companions only outside the completeness region are considered as single stars. Therefore the restricted binary star frequency is a lower limit to the true binary star frequency. Nonetheless, the restricted binary star frequency of this sample is well defined and is therefore useful for the comparisons listed in Table 6 and discussed below.

\subsection{Binary Stars and Star Forming Regions}

One of the goals of this study was to investigate the physical processes that control the formation of multiple
TABLE 5(a). Limits for stars observed as multiple systems in Oph-Sco.

\begin{tabular}{lcccc}
\hline \hline \multicolumn{1}{c}{ Object } & Date & \multicolumn{3}{c}{ Flux Ratio Limits } \\
& & $0 . " 10$ & $0 . " 20$ & $0 . " 80$ \\
\hline SR 20 & 1990 July 9 & $28 \pm 5$ & $30 \pm 6$ & $30 \pm 6$ \\
SR 9 & 1990 Aug 5 & $24 \pm 7$ & $26 \pm 8$ & $27 \pm 8$ \\
& 1990 July 9 & $16 \pm 3$ & $16 \pm 3$ & $16 \pm 3$ \\
ROX 42C & 1991 May 3 & $21 \pm 4$ & $23 \pm 5$ & $23 \pm 5$ \\
& 1990 July 8 & $13 \pm 4$ & $13 \pm 4$ & $13 \pm 4$ \\
Haro 1-4 & 1990 July 9 & $21 \pm 4$ & $21 \pm 4$ & $24 \pm 6$ \\
160946-1851 & 1990 Aug 5 & $19 \pm 3$ & $33 \pm 9$ & $33 \pm 9$ \\
162218-2420 & 1990 July 8 & $19 \pm 4$ & $20 \pm 5$ & $20 \pm 5$ \\
DoAr 24 E & 1990 July 9 & $19 \pm 4$ & $19 \pm 4$ & $19 \pm 4$ \\
155293-2338 & 1990 July 8 & $9 \pm 2$ & $9 \pm 2$ & $12 \pm 3$ \\
AS 205 & 1990 July 7 & $7 \pm 1$ & $8 \pm 1$ & $8 \pm 1$ \\
V853 Oph & 1990 Aug 7 & $7 \pm 1$ & $7 \pm 1$ & $8 \pm 2$ \\
155913-2233 & 1990 July 8 & $5.9 \pm 0.8$ & $10 \pm 2$ & $12 \pm 3$ \\
\hline \hline
\end{tabular}

TABLE 5(b). Limits for stars observed as multiple systems in Tau-Aur.

\begin{tabular}{llccc}
\hline \hline \multicolumn{1}{c}{ Object } & \multicolumn{1}{c}{ Date } & \multicolumn{3}{c}{ Flux Ratio Limits } \\
& & $0 . " 10$ & $0 . " 20$ & $0 . " 80$ \\
\hline T Tau & 1991 Nov 18 & $56 \pm 18$ & $56 \pm 18$ & $56 \pm 18$ \\
DD Tau & 1990 Oct 4 & $42 \pm 10$ & $42 \pm 10$ & $42 \pm 10$ \\
& 1990 Oct 2 & $20 \pm 5$ & $21 \pm 6$ & $23 \pm 7$ \\
RW Aur & 1990 Nov 9 & $41 \pm 13$ & $41 \pm 13$ & $41 \pm 13$ \\
UY Aur & 1990 Nov 10 & $33 \pm 10$ & $36 \pm 12$ & $39 \pm 14$ \\
V807 Tau & 1990 Nov 10 & $25 \pm 4$ & $47 \pm 13$ & $47 \pm 13$ \\
FO Tau & 1991 Oct 18 & $23 \pm 5$ & $34 \pm 9$ & $34 \pm 9$ \\
UZ Tau W & 1990 Nov 10 & $23 \pm 7$ & $23 \pm 7$ & $27 \pm 10$ \\
V928 Tau & 1990 Nov 9 & $20 \pm 6$ & $26 \pm 9$ & $26 \pm 9$ \\
DF Tau & 1990 Nov 9 & $20 \pm 5$ & $20 \pm 5$ & $20 \pm 5$ \\
DI Tau & 1991 Oct 18 & $17 \pm 4$ & $18 \pm 5$ & $19 \pm 5$ \\
LkH $\alpha 332 / G 1$ & 1990 Oct 4 & $17 \pm 3$ & $59 \pm 24$ & $59 \pm 24$ \\
& 1990 Oct 2 & $7 \pm 1$ & $12 \pm 3$ & $12 \pm 3$ \\
XZ Tau & 1991 Oct 20 & $15 \pm 4$ & $15 \pm 4$ & $15 \pm 4$ \\
IS Tau & 1991 Oct 19 & $13 \pm 3$ & $16 \pm 4$ & $17 \pm 4$ \\
GG Tau & 1991 Oct 20 & $12 \pm 3$ & $12 \pm 3$ & $13 \pm 3$ \\
FX Tau & 1991 Oct 18 & $12 \pm 3$ & $12 \pm 3$ & $12 \pm 3$ \\
V410 Tau & 1991 Oct 20 & $12 \pm 3$ & $16 \pm 5$ & $16 \pm 5$ \\
FV Tau & 1991 Oct 20 & $10 \pm 2$ & $15 \pm 5$ & $16 \pm 5$ \\
V773 Tau & 1990 Oct 3 & $10 \pm 2$ & $10 \pm 2$ & $10 \pm 2$ \\
LkCa 3 & 1991 Oct 18 & $9 \pm 2$ & $9 \pm 2$ & $9 \pm 2$ \\
GH Tau & 1991 Oct 19 & $7.8 \pm 0.8$ & $7.8 \pm 0.8$ & $7.8 \pm 0.8$ \\
DK Tau & 1991 Oct 20 & $5 \pm 2$ & $5 \pm 2$ & $5 \pm 2$ \\
\hline \hline
\end{tabular}

star systems. The large fraction of $\mathrm{T}$ Tauri stars observed with at least one companion star suggests that multiple stars are formed during the process of star formation, as opposed to being formed at some later time, e.g., by capture (see also discussion below). Thus the various physical and environmental conditions of the regions where these stars originate are presumably among the factors that effect binary star formation. This section examines the multiplicity of $\mathrm{T}$ Tauri stars as a function of star forming region, in an attempt to understand what environmental factors affect binary star formation.

The restricted binary star frequency of the complete sample provides the basis for comparing the binary star frequencies of the two star forming regions, Tau-Aur and Oph-Sco. In the completeness region, 6 out of 21 stars in Oph-Sco $(29[ \pm 12] \%)$ and 16 out of 43 stars in Tau-Aur $(37[ \pm 9] \%)$ have companion stars. Figure 9 shows the 

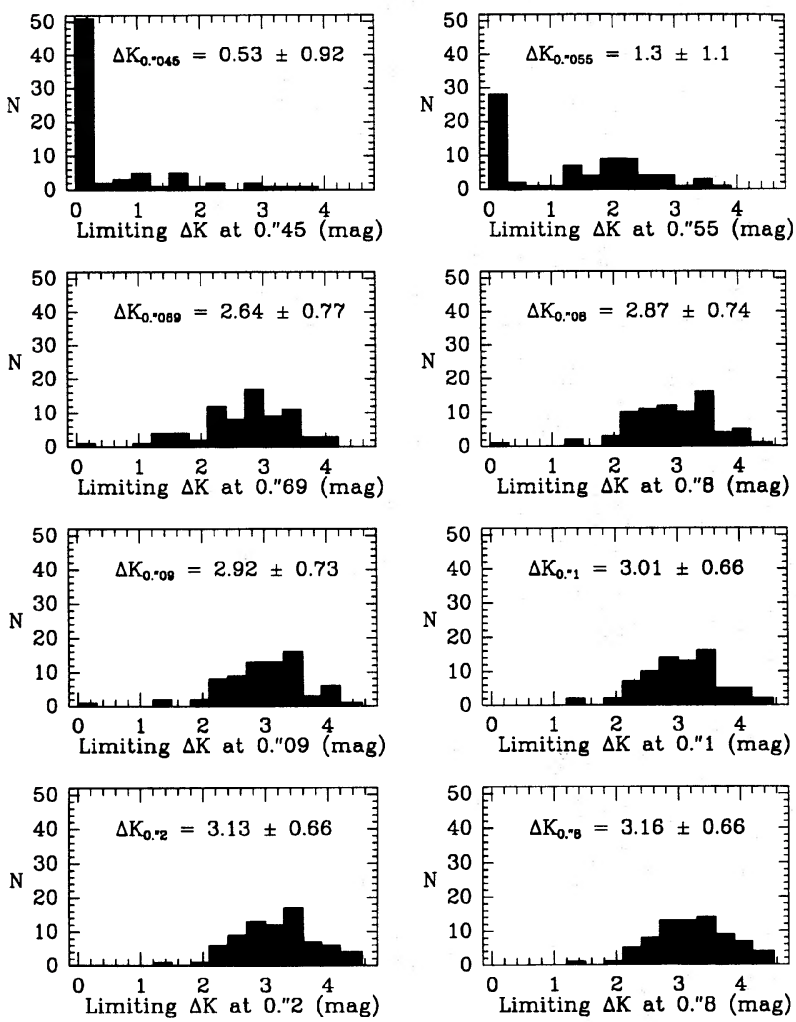

FIG. 5. Histograms of the limiting $K$ magnitude differences for unobserved companions at $0.045,0 " 055,0.069,0.08,0$."1, 0".2, and $0^{\prime \prime} 8$. Each histogram contains a total of 69 limits, one for each target. In the cases of repeated observations of a target star the best limits were used.

restricted binary star frequency for the two regions as a function of separation; they appear to be quite similar. The result of a Kolmogorov-Smirnov test indicates a 0.991 probability that the two distributions are the same as a

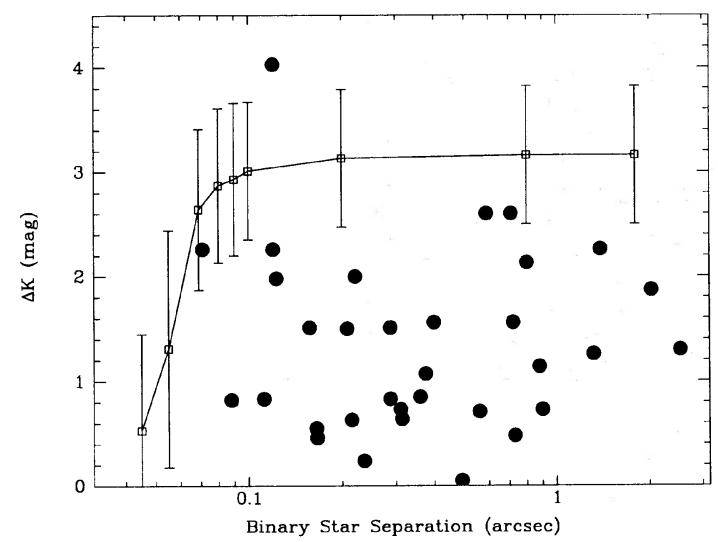

FIG. 6. The average sensitivity curve, measured in terms of limiting $K$ magnitude difference, is plotted as a function of separation. Also plotted here are the observed binary star magnitude differences. On average the observations are of sufficient quality to detect binary stars with magnitude difference of up to 3.1 for separations between 0.1 and 1 ".8.

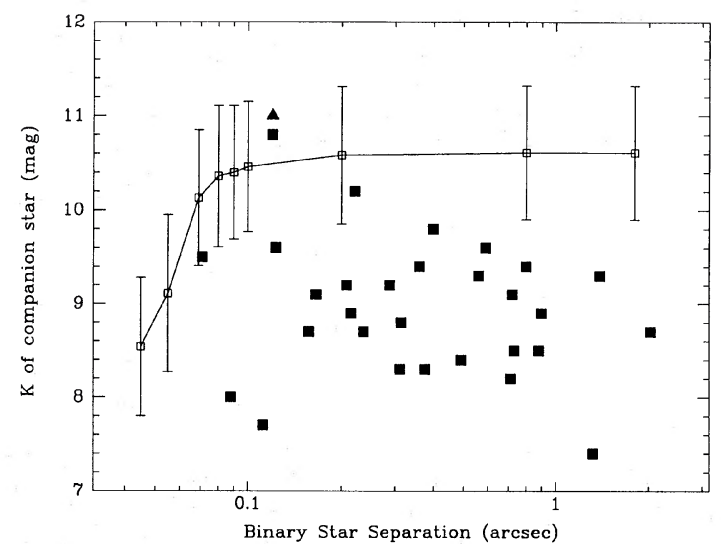

FIG. 7. The average sensitivity curve, measured in terms of limiting apparent $K$ magnitude of a companion star, is plotted as a function of separation. Also plotted here are the observed companion star magnitudes. Secondaries are plotted as squares and the one tertiary is plotted as a triangle. On average the observations are of sufficient quality to detect companion stars brighter than $K=10.6$ mag at separations between 0 ". 1 and 1.8 .

function of separation between 16 and $252 \mathrm{AU}$. Thus there is no statistically significant difference between the binary star frequencies of the two star forming regions over the projected linear separation range 16 to $252 \mathrm{AU}$.

Tau-Aur and Oph-Sco are alike in that they are both sites of low mass star formation, contain dark clouds (Lynds 1962), and have a total mass of $\approx 10^{4} \mathscr{M} \odot$ (de Geus et al. 1990; Ungerechts \& Thaddeus 1987). These sites, however, have distinct differences. One distinction between these regions is the presence of a large centrally condensed core and an associated high density of young stellar objects in Oph-Sco. This high mass core (the $\rho$ Oph cloud) appears to have a much higher star forming efficiency (Wilking \& Lada 1983; Wilking et al. 1989) than the low mass cloud cores which are distributed throughout both regions and are characterized by much lower stellar densities and lower overall star formation efficiency (Myers 1985).

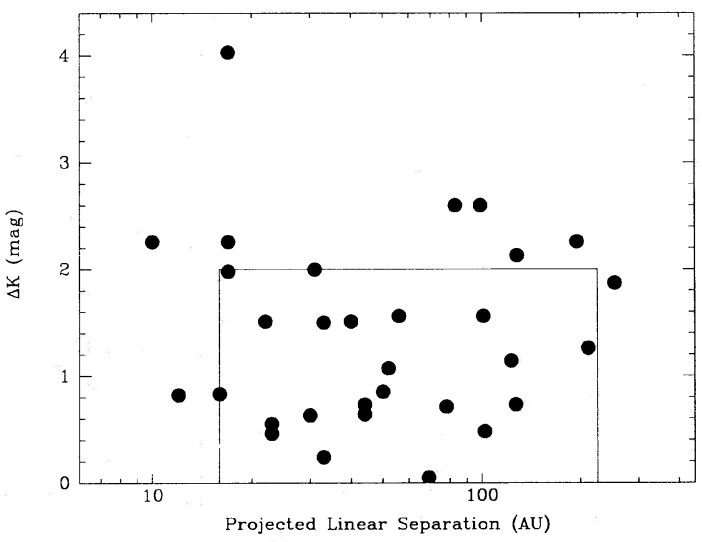

FIG. 8. The distribution of the observed binary stars (circles) and the limits of the completeness region (the outlined area). 
TABLE 6. Multiplicity of T Taur stars in the complete sample. ${ }^{a}$

\begin{tabular}{lcccc}
\hline \hline Sample & \# Targets & $\begin{array}{c}\text { \# Companions in } \\
\text { completeness region }\end{array}$ & $\begin{array}{c}\text { bsf }^{\mathrm{b}} \\
(\%)\end{array}$ & Section \\
\hline Total & 64 & 22 & $34 \pm 7$ & 5.1 \\
Oph-Sco & 21 & 6 & $29 \pm 12$ & 5.2 \\
Tau-Aur & 43 & 16 & $37 \pm 9$ & \\
WTTS & 22 & 8 & $36 \pm 13$ & 5.3 \\
CTTS & 42 & 14 & $33 \pm 9$ & \\
$\mathscr{M}<1 \mathscr{M}_{\odot}$ & 32 & 13 & $41 \pm 11$ & 5.4 \\
$\mathscr{M}>1 \mathscr{M}_{\odot}$ & 32 & 9 & $28 \pm 9$ & \\
\hline \hline
\end{tabular}

${ }^{a}$ The complete sample, discussed in Sec. 5.1, includes all observations sensitive to the "completeness region," i.e., that revealed all companion stars within the projected linear separation range 16 to $252 \mathrm{AU}$ and within the magnitude difference range 0 to $2.0 \mathrm{mag}$.

${ }^{b}$ The restricted binary star frequency (bsf) incorporates only companion stars within the completeness region, and is therefore a lower limit to the true binary star frequency in the separation range 16 to 252 AU. Nonetheless, it is useful for comparisons of various groups of T Tauri stars, which are discussed in the sections listed in Column 5.

Lada et al. (1992) suggest, on the basis of these differences, that two modes of, or environments for, star formation exist: isolated, i.e., in the scattered low mass cloud cores, and clustered, i.e., in the high mass cloud cores. In the complete sample, the restricted binary star frequency of the $\rho$ Oph cloud core $(44[ \pm 22] \%, 9$ stars $)$ is not significantly different from that of Tau-Aur, which is composed of low mass cloud cores.

Five of the T Tauri stars in the Oph-Sco complete sample are members of Sco OB2, an OB association. These stars and those in Oph are thought to be produced from the same giant molecular cloud material (Blaauw 1964; de Geus et al. 1989), where the formation of the OB association was triggered at an earlier time then that of the low mass stars in Oph. The restricted binary star frequency of the Sco OB2 sample $(40[ \pm 28] \%)$ is not statistically different from that of the remainder of the complete sample

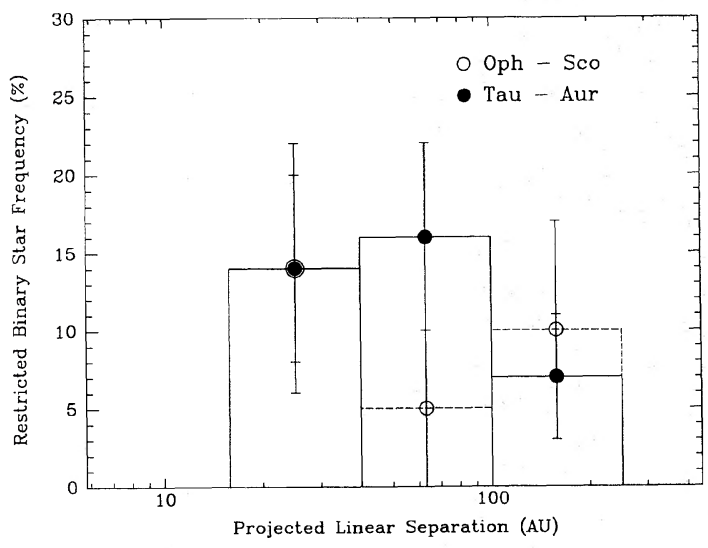

FIG. 9. The binary star frequency as a function of projected linear separation for Tau-Aur (filled circles) and Oph-Sco (unfilled circles). There appears to be no statistically significant difference in the overall binary star frequency between these two star forming regions.

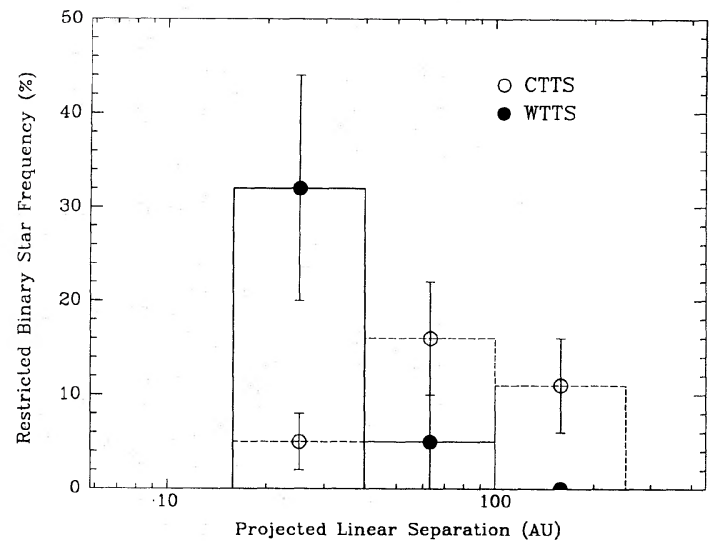

FIG. 10. The binary star frequency as a function of projected linear separation for the WTTS (filled circles) and the CTTS (unfilled circles). The WTTS dominate the binary star distribution at shorter separations whereas the CTTS populate the wider binary star separations.

$(34[ \pm 8] \%)$. However, neither of these last two comparisons is very sensitive due to the small number of observations in the $\rho$ Oph cloud core and Sco OB2, respectively.

\subsection{Binary Stars and Circumstellar Disks}

Another aim of this survey was to examine the physical relationship between the two observationally defined subclasses of the $T$ Tauri stars, the classical $T$ Tauri stars (CTTS) and the weak-lined T Tauri stars (WTTS). These two subgroups differ in their $\mathrm{H} \alpha$ emission: CTTS have strong and broad $\mathrm{H} \alpha$ emission $[W(\mathrm{H} \alpha)>10 \AA]$ whereas WTTS have weak and narrow $\mathrm{H} \alpha$ emission $[W(\mathrm{H} \alpha)<10$ Å] (e.g., Herbig \& Bell 1988; Strom et al. 1989). Most researchers agree that the weak $\mathrm{H} \alpha$ emission observed in WTTS probably originates from the chromosphere, whereas many theories have been presented to account for the presence of strong $\mathrm{H} \alpha$ emission in CTTS. A recent and intriguing proposal suggests that the strong $\mathrm{H} \alpha$ emission arises from disk accretion (Basri \& Bertout 1989).

The results of this survey provide an opportunity to investigate the possibility that the underlying difference between the WTTS and the CTTS is related to the presence of a close secondary star. In the completeness region, 14 of the 42 CTTS $(33[ \pm 9] \%)$ and 8 of the 22 WTTS (36 $[ \pm 13] \%$ ) have companion stars; the binary star frequency appears to be similar in the two subclasses. However, integration over the separation range 16 to $252 \mathrm{AU}$ could have washed out any effect due to circumstellar disks, since such disks are typically assumed to be roughly $100 \mathrm{AU}$ in size. Figure 10 shows the CTTS and WTTS restricted binary star frequency binned as a function of projected linear separation. There is an apparent difference in the dependence on separation; the WTTS populate the binary star distribution at the closer separations and the CTTS dominate the distribution at wider separations. A KolmogorovSmirnov test on the data indicates a probability of 0.009 for the two sets being drawn from the same distribution. Thus 
the distributions of the CTTS and WTTS binary stars as a function of separation are significantly different from each other. The WTTS binary star distribution is enhanced at smaller separations relative to the CTTS binary star distribution.

The observed difference between the CTTS and WTTS binary star distributions suggests that close companion stars play a significant role in the differentiation of these two T Tauri star subclasses. We adopt Basri \& Bertout's (1989) model, in which the strong $\mathrm{H} \alpha$ emission of the CTTS arises from the boundary layer of an accretion disk. It has been suggested that all T Tauri stars are formed with an accretion disk (e.g., Shu et al. 1987) and therefore would be initially classified as CTTS; they would be observed as WTTS only when their accretion disks are depleted. Walter et al. (1988) conclude that the two subclasses are more or less coeval, which suggests that the timescale for disk dispersal depends on more than just the age or mass of the parent $T$ Tauri star. It should be noted, however, that the very oldest population of $\mathrm{T}$ Tauri stars appears as WTTS (Walter et al.).

A close secondary star can create a gap in the circumstellar disk (Lin \& Papaloizou 1992), truncating the amount of material available for accretion. We propose that by reducing the amount of material that can be accreted, the effect of a close secondary star is to shorten the accretion time scale in close binary $T$ Tauri stars. In this way those $\mathrm{T}$ Tauri stars with close companions would quickly deplete their accretion disks and appear as WTTS at a fairly early age. Presumably, secondary stars outside the circumstellar disk ( $>\sim 100 \mathrm{AU}$ ) should be of no consequence to the accretion time scale.

\subsection{Binary Stars and Mass}

In theory, $\mathrm{T}$ Tauri stars evolve to the main sequence along convective-radiative tracks for low mass stars $\left(\mathscr{M}<3.0 \mathscr{M}_{\odot}\right)$ such as those given by Cohen \& Kuhi (1979) and reproduced in Fig. 11. Since the evolutionary tracks for low mass stars are fairly constant in effective temperature, much can be learned just from the spectral types of the stars. Table 7 lists the spectral types of the T Tauri stars in the complete sample and Fig. 12(a) displays their distribution with spectral type. The restricted binary star frequency, shown in Fig. 12(b), suggests a dependence on spectral type. Since the later spectral types in general correspond to lower masses, there may be a dependence on mass.

Mass estimates for these stars can be obtained from their location in the HR diagram with respect to the evolutionary tracks. This requires both the effective temperature, $T_{\text {eff }}$, and stellar luminosity, $L$, for each star. The effective temperatures were derived from the spectral types using the conversion adopted by Cohen \& Kuhi (1979). Column 6 of Table 7 lists the stellar luminosities estimated from optical and near-infrared data. These values are used directly for the target stars observed without companion stars. For those stars observed with companions, these luminosities are overestimates due to the extra flux from the

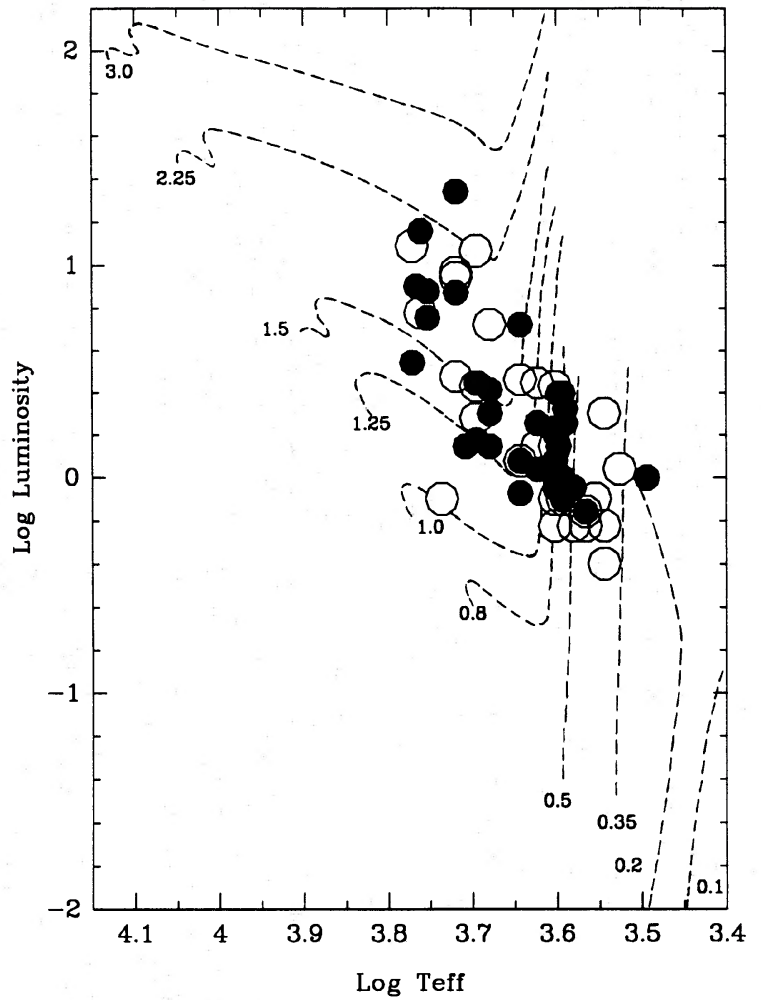

FIG. 11. HR diagram for the stars in the sample. Also plotted are the evolutionary tracks from Cohen \& Kuhi (1979). The filled circles are stars observed without companion stars. The unfilled points are those stars observed with companions (the estimated contribution from the companion stars has been removed from the luminosity of these stars).

secondary stars. The brightest component observed at $K$ is assumed to be the optically identified $T$ Tauri star, for which the spectral type is known, and the measured $K$ flux density ratio is used to divide the previously derived stellar luminosity between the components of the system.

Figure 11 shows the stars in the complete sample in an HR diagram along with the evolutionary tracks from Cohen \& Kuhi (1979). The estimated masses for these T Tauri stars range from 0.2 to $2.3 \mathscr{M}_{\odot}$ and the distribution peaks between 0.5 and $1.5 \mathscr{M}_{\odot}$. Figure 13 demonstrates the mass dependence on effective temperature, or equivalently spectral type. By separately grouping spectral types M5 through K7 and K6 through G0, the restricted binary star frequency for $\mathrm{T}$ Tauri stars with $\leqslant 1 \mathscr{M}_{\odot}$ can be compared to that of stars with masses $\geqslant 1 \mathscr{M}_{\odot}$. In the completeness region, 13 of 32 stars with $\mathscr{M}<\mathscr{M}_{\odot}(41[ \pm 11] \%)$ and 9 of 32 stars with $\mathscr{M} \geqslant 1 \mathscr{M}_{\odot}(28[ \pm 9] \%)$ have companion stars. Although the lower mass stars in the sample have a higher restricted binary star frequency, the difference is not statistically significant. Furthermore, care must be taken in comparing the restricted binary star frequencies of different mass stars. Given the shape of the convective-radiative tracks and the isochrones, magnitude differences most likely reflect mass differences. If the lower mass stars do indeed have a higher restricted binary star frequency, that 
would most likely indicate that the lower mass have more high mass ratio (i.e., $\mathscr{M}_{1} / \mathscr{M}_{2}$ tends toward one) binary systems than the higher mass targets. Such a distinction would provide a strong constraint on star formation mechanisms.

\subsection{T Tauri Versus Main Sequence Binary Stars}

The results of this survey indicate that a large fraction of $\mathrm{T}$ Tauri stars have companion stars. The youth of these stars $\left(<\sim 10^{7} \mathrm{yr}\right)$ suggests that multiple star systems are produced during the early stages of star formation.

To compare the binary star frequency of the $T$ Tauri stars to that of the main-sequence stars, multiplicity surveys of main-sequence stars that have a mass range similar to that of the T Tauri stars were selected. Duquennoy \& Mayor (1991) surveyed a sample of nearby solar type main-sequence stars with spectral types F7 to G9. This corresponds to a range in main-sequence masses of $\sim 0.8$ to $1.3 \mathscr{M}_{\odot}$. Therefore this sample of main-sequence stars appears to be an appropriate older counterpart for a large percentage (44\%) of the T Tauri sample. Fischer \& Marcy (1992) investigated the multiplicity of the nearby mainsequence $\mathrm{M}$ dwarfs which have masses between $\sim 0.1$ and $\sim 0.5 \mathscr{M}_{\odot}$. This mass range incorporates $28 \%$ of the $\mathrm{T}$ Tauri stars in the complete sample and therefore Fischer and Marcy's study is also useful for comparison with the $T$ Tauri results.

The main-sequence surveys mentioned above combine the results of many detection techniques and therefore cover a much larger binary star separation range than the speckle imaging survey of $\mathrm{T}$ Tauri stars. Consequently a comparison between these samples requires a calculation of the binary star frequency for the main-sequence surveys in the projected linear separation range 16 to $252 \mathrm{AU}$; see Appendix 1.

Since the M dwarf and solar type (F7 to G9) mainsequence stars have similar binary star frequencies in the projected linear separation range 16 to $252 \mathrm{AU}, 12[ \pm 4] \%$ and $16[ \pm 3] \%$, respectively, and the solar-type mainsequence stars are the older counterparts to the majority of the $\mathrm{T}$ Tauri stars, the latter is used for comparison with the $\mathrm{T}$ Tauri binary star frequency.

The main-sequence results are corrected for detection biases and therefore provide estimates of the mainsequence binary star frequency integrated over all magnitude differences. Thus the estimate of the binary star frequency in the projected linear separation range 16 to 252

TABLE 7(a). Stars in the complete sample observed without companion stars.

\begin{tabular}{|c|c|c|c|c|c|c|c|}
\hline HBC & Name & SpT & Ref & $\begin{array}{c}\log \mathrm{T}_{\text {eff }} \\
(K)\end{array}$ & $\begin{array}{l}\mathrm{L}^{*} \\
\left(L_{O}\right)\end{array}$ & Ref & $\begin{array}{l}\text { Mass } \\
\left(M_{O}\right)\end{array}$ \\
\hline 259 & SR 4 & K6 & 2 & 3.623 & 1.8 & 2 & 1.25 \\
\hline 262 & SR $24 \mathrm{~S}$ & K2 & 1 & 3.695 & 2.8 & 1 & 1.5 \\
\hline 267 & Haro 1-14 & M0 & 1 & 3.593 & 1.0 & 1 & 0.8 \\
\hline 268 & Haro 1-16 & K3 & 1 & 3.679 & 1.4 & 2 & 1.25 \\
\hline 270 & V1121 Oph & K5 & 1 & 3.643 & 5.3 & 1 & 1.6 \\
\hline 637 & DoAr 21 & K0 & 2 & 3.719 & 22.0 & 2 & 2.5 \\
\hline 638 & DoAr 24 & K5 & 2 & 3.643 & 1.2 & 2 & 1.25 \\
\hline \multirow[t]{4}{*}{649} & RNO 90 & G5 & 6 & 3.753 & 5.7 & 16 & 1.6 \\
\hline & $160815-1857$ & $\mathrm{~K} 2$ & 17 & 3.695 & 1.5 & 17 & 1.3 \\
\hline & $160827-1813$ & K5 & 17 & 3.643 & 0.8 & 17 & 1.2 \\
\hline & ROXs 43A & G0 & 2 & 3.771 & 3.5 & 2 & 1.35 \\
\hline 24 & FN Tau & M5 & 1 & 3.494 & 1.0 & 8 & 0.2 \\
\hline 25 & CW Tau & K3 & 1 & 3.679 & 2.6 & 8 & 1.55 \\
\hline 28 & CY Tau & M1 & 1 & 3.566 & 0.7 & 8 & 0.45 \\
\hline 32 & BP Tau & K7 & 1 & 3.602 & 1.2 & 8 & 0.8 \\
\hline 34 & RY Tau & K0 & 3 & 3.719 & 7.5 & 8 & 1.9 \\
\hline 37 & DG Tau & K7 & 3 & 3.602 & 1.7 & 8 & 0.8 \\
\hline 41 & IQ Tau & M0.5 & 1 & 3.580 & 0.9 & 8 & 0.5 \\
\hline 49 & HL Tau & K7 & 3 & 3.602 & 0.9 & 8 & 0.8 \\
\hline 52 & UZ Tau E & M0 & 3 & 3.593 & 0.8 & 13 & 0.7 \\
\hline 56 & GI Tau & K7 & 1 & 3.602 & 1.1 & 7 & 0.8 \\
\hline 57 & GK Tau & K7 & 1 & 3.602 & 1.6 & 8 & 0.8 \\
\hline 61 & CI Tau & K6 & 3 & 3.623 & 1.1 & 7 & 1.1 \\
\hline 66 & HP Tau & K3 & 1 & 3.679 & 2.0 & 8 & 1.4 \\
\hline 67 & DO Tau & K7-M0 & 1 & 3.598 & 1.4 & 8 & 0.75 \\
\hline 74 & DR Tau & M0 & 3 & 3.593 & 2.5 & 8 & 0.75 \\
\hline 79 & SU Aur & G2 & 3 & 3.761 & 14.4 & 8 & 2.15 \\
\hline 374 & Hubble 4 & K7-M0 & 1 & 3.598 & 2.5 & 16 & 0.8 \\
\hline 378 & V819 Tau & K7 & 10 & 3.602 & 1.0 & 8 & 0.8 \\
\hline 380 & HDE 283572 & G5 & 10 & 3.753 & 7.6 & 13 & 1.8 \\
\hline 388 & $042417+1744$ & K1 & 10 & 3.708 & 1.4 & 13 & 1.25 \\
\hline 396 & Haro 6-13 & Cont & 1 & 3.590 & 2.1 & 8 & 0.7 \\
\hline 402 & FZ Tau & Cont & 1 & 3.590 & 1.8 & 14 & 0.7 \\
\hline 415 & HP Tau/G2 & G1 & 1 & 3.766 & 8.0 & 13 & 1.8 \\
\hline
\end{tabular}

References contained in notes following Table $7 \mathrm{~b}$. 
TABLE 7(b). Stars in the complete sample observed with companion stars.

\begin{tabular}{|c|c|c|c|c|c|c|c|c|}
\hline HBC & Name & SpT & Ref & $\begin{array}{c}\log \mathrm{T}_{\text {eff }} \\
(K)\end{array}$ & $\begin{array}{l}L_{*}^{\text {old }} \\
\left(L_{O}\right)\end{array}$ & Ref & $\begin{array}{l}L^{\text {prim }} \\
\left(L_{O}\right)\end{array}$ & $\begin{array}{l}\text { Mass } \\
\left(M_{O}\right)\end{array}$ \\
\hline 254 & AS 205* & $\mathrm{K} 2$ & 3 & 3.695 & 15.3 & 1 & 11.7 & 2.25 \\
\hline 257 & Haro 1-4* & K6 & 1 & 3.623 & 3.5 & 1 & 2.8 & 1.25 \\
\hline 264 & SR 9 & K7 & 1 & 3.602 & 0.9 & 2 & 0.8 & 0.85 \\
\hline 266 & V853 Oph* & M1.5 & 1 & 3.555 & 1.0 & 1 & 0.8 & 0.45 \\
\hline 639 & DoAr $24 \mathrm{E}$ & K0 & 2 & 3.719 & 11.0 & 2 & 9.3 & 2.0 \\
\hline \multirow[t]{5}{*}{643} & SR 20 & G0 & 2 & 3.771 & 13.9 & 2 & 12.3 & 2.0 \\
\hline & $155203-2338$ & G2 & 17 & 3.761 & 6.8 & 17 & 6.0 & 1.8 \\
\hline & $160946-1851^{*}$ & K0 & 17 & 3.719 & 3.8 & 17 & 3.0 & 1.5 \\
\hline & $162218-2420 *$ & G8 & 5 & 3.736 & 1.5 & 16 & 0.8 & 1.0 \\
\hline & ROXs $42 C^{*}$ & K6 & 2 & 3.623 & 1.8 & 2 & 1.4 & 1.25 \\
\hline 29 & V410 Tau* & K2 & 3 & 3.695 & 2.2 & 8 & 1.9 & 1.35 \\
\hline 30 & DD Tau* & M1 & 1 & 3.566 & 1.0 & 8 & 0.7 & 0.45 \\
\hline 35 & T Tau & K0 & 3 & 3.719 & 8.8 & 15 & 8.8 & 2.0 \\
\hline 36 & DF Tau & M2 & 3 & 3.544 & 3.0 & 8 & 2.0 & 0.4 \\
\hline 39 & DI Tau & M0 & 1 & 3.593 & 0.9 & 8 & 0.8 & 0.7 \\
\hline 44 & FX Tau* & M1 & 1 & 3.566 & 1.0 & 8 & 0.7 & 0.45 \\
\hline 50 & XZ Tau* & M3 & 1 & 3.526 & 1.6 & 8 & 1.1 & 0.35 \\
\hline 53 & UZ Tau W* & M2 & 3 & 3.544 & 0.6 & 14 & 0.4 & 0.4 \\
\hline 54 & GG Tau* & K7 & 3 & 3.602 & 1.8 & 7 & 1.4 & 0.8 \\
\hline 55 & GH Tau* & M2 & 1 & 3.544 & 0.9 & 8 & 0.6 & 0.4 \\
\hline 59 & IS Tau* & K2 & 1 & 3.695 & 3.1 & 8 & 2.7 & 1.5 \\
\hline 76 & UY Aur* & K7 & 3 & 3.602 & 0.8 & 7 & 0.6 & 0.75 \\
\hline 80 & RW Aur & K5 & 3 & 3.623 & 3.3 & 14 & 2.9 & 1.4 \\
\hline 367 & V773 Tau* & K3 & 9 & 3.679 & 7.7 & 8 & 5.3 & 1.8 \\
\hline 368 & LkCa 3* & M1 & 11 & 3.566 & 1.3 & 12 & 0.7 & 0.45 \\
\hline 369 & FO Tau* & M2 & 1 & 3.544 & 1.0 & 14 & 0.6 & 0.4 \\
\hline 386 & FV Tau* & K5 & 1 & 3.643 & 2.0 & 14 & 1.2 & 1.0 \\
\hline 398 & V928 Tau* & M0.5 & 1 & 3.580 & 1.0 & 1 & 0.6 & 0.5 \\
\hline 404 & V807 Tau* & K7 & 4 & 3.602 & 3.7 & 16 & 2.7 & 0.8 \\
\hline 423 & $\mathrm{LkH} \alpha 332 / \mathrm{G} 1^{*}$ & M1 & 1 & 3.566 & 0.9 & 14 & 0.6 & 0.45 \\
\hline
\end{tabular}

Notes: $\left(^{*}\right)$ denotes binary stars that fall within the completeness region, i.e., have separations in the range 16 to $252 \mathrm{AU}$ and magnitudes differences between 0.0 and $2.0 \mathrm{mag}$.

References for Column 4 (Spectral Type) and Column 7 (Stellar Luminosity). Also noted are the assumed distances to the star forming regions used in the references, which derive stellar luminosities. The stellar luminosities listed in Column 6 have been shifted, when necessary for the distances assumed in this work $\left(d_{\text {Tau-Aur }}=140 p c, d_{O p h}=140 p c\right.$, and $\left.d_{S c o}=160 p c\right)$.

(1) Cohen \& Kuhi (1979); $d_{\text {Tau }}=160 p c ; d_{O p h}=170 p c$

(2) Bouvier \& Appenzeller (1991); $d_{O p h}=170 p c$

(3) Basri \& Batalha (1990)

(4) Herbig \& Bell (1988)

(5) Montmerle et al. (1983)

(6) Herbst \& Warner (1981)

(7) Hartigan et al. (1991); $d_{\text {Tau }}=160 p c$

(8) Beckwith et al. (1990); $d_{T a u}=140 p c$

(9) Herbig (1977)
(10) Walter et al. (1988)

(11) Herbig et al. (1986)

(12) Cabrit et al. (1990); $d_{\text {Tau }}=160 p c$

(13) Strom et al. (1989); $d_{\text {Tau }}=160 p c$

(14) Cohen et al. (1989); $d_{\text {Tau }}=160 p c$

(15) Ghez et al. (1991); $d_{T a u}=140 p c$

(16) Simon et al. (1992)

(17) Walter et al. (1993)
AU for the $\mathrm{T}$ Tauri stars must include all magnitude differences,

$$
\mathrm{BSF}^{\prime}=\int_{0}^{\infty} \int_{16}^{252} \frac{d \mathrm{BSF}}{d s d \Delta K} d s d \Delta K .
$$

An estimate of $\mathrm{BSF}^{\prime}$ requires a calculation of the distribution, in $\Delta K$, of the binary star frequency for $\Delta K>2.0 \mathrm{mag}$ (bsf already accounts for $\Delta K \leqslant 2.0 \mathrm{mag}$ ). This was accomplished by examining strips in the $\Delta K$-projected linear separation parameter space above $\Delta K=2.0 \mathrm{mag}$. For each $\Delta K$ strip, spanning 16 to $252 \mathrm{AU}$ and an interval of $0.5 \mathrm{mag}$ in magnitude difference, the number of observations sensitive to this region and, of these, how many have companion stars that fall within that region were determined (see Table 8 ). There are observations sensitive to magnitude dif- ferences of 4.0 mag; beyond this range there is no way of estimating the binary star frequency. Given these uncertainties, the estimated binary star frequency, as derived from these results in the projected linear separation range 16 to $252 \mathrm{AU}$ is $59[ \pm 17] \%$.

In both the main sequence and the $\mathrm{T}$ Tauri study the total number of target stars in each was used to normalize the binary star frequency. However, Duquennoy \& Mayor's (1991) definition of a target star differs from the one used in this study in the question of how far another star could be before it is considered to be a separate target star. For this discussion the lowest value, $2 \times 10^{3} \mathrm{AU}$, suggested by Duquennoy and Mayor for the cutoff separation is adopted. This affects only the treatment of UZ Tau E and $\mathrm{W}$, which counted previously as two targets and are now 

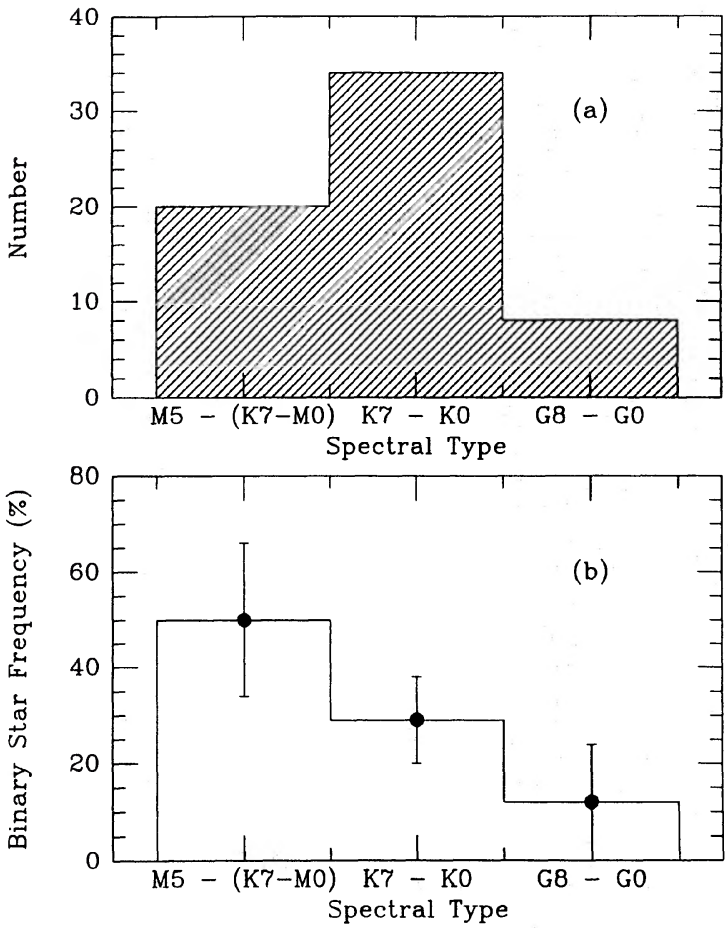

FIG. 12. (a) The distribution of spectral types for the complete sample. (b) The restricted binary star frequency as a function of spectral type.

viewed as one system. This only slightly changes the value for the $\mathrm{T}$ Tauri binary star frequency in the projected linear separation range 16 to $252 \mathrm{AU}$, which was previously estimated to $59[ \pm 17] \%$ and is now taken to be $60[ \pm 17] \%$.

The $T$ Tauri binary star frequency appears to be greater than that of the main-sequence stars by a factor of 4 [see Fig. 14(b)]. The discrepancy between the two binary star frequencies is a $2.5 \sigma$ effect.

The discrepancy between the two binary star frequencies could be due to a difference in the sensitivity of the two surveys, which would imply that this survey is much more

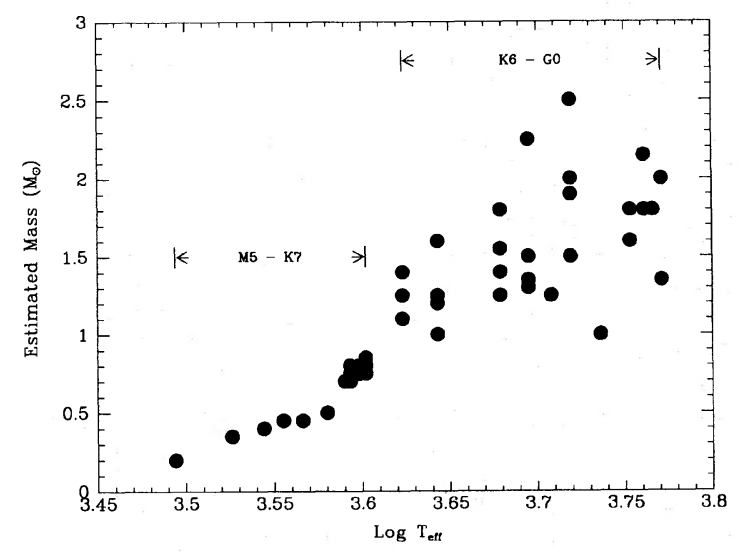

FIG. 13. The estimated mass as a function of effective temperature (or equivalently spectral type).
TABLE 8. T Tauri binary stars frequency.

\begin{tabular}{cccr}
\hline $\begin{array}{c}\Delta \mathrm{K} \text { Range } \\
(\mathrm{mag})\end{array}$ & \# Obs & \# Companions & \multicolumn{1}{c}{$\begin{array}{c}\text { Freq } \\
(\%)\end{array}$} \\
\hline $0.0-2.0$ & 64 & 22 & $34 \pm 7$ \\
$2.0-2.5$ & 54 & 3 & $6 \pm 4$ \\
$2.5-3.0$ & 38 & 2 & $5 \pm 4$ \\
$3.0-3.5$ & 15 & 0 & 0 \\
$3.5-4.0$ & 7 & 1 & $14 \pm 14$ \\
\hline $0.0-4.0$ & $\ldots$ & $\ldots$ & $59 \pm 17$ \\
\hline
\end{tabular}

sensitive to companion stars than Duquennoy \& Mayor's (1991) survey. However, the solar-type main-sequence sample has already been corrected for undetected companion stars down to visual magnitude differences of $7.0 \mathrm{mag}$, whereas the $T$ Tauri results are sensitive to companions with magnitude difference of at most 4.0 mag. Although it is difficult to determine how $\Delta K$ 's for $\mathrm{T}$ Tauri stars correspond to $\Delta V$ 's for main-sequence stars, it seems unlikely that the apparent overabundance of companion stars among the $T$ Tauri stars compared to the solar-type mainsequence stars is the result of undetected companions in Duquennoy and Mayor's sample.

This $\mathbf{T}$ Tauri star survey is magnitude limited, which makes it possible for the binary star frequency to be artificially high if the inclusion of the companions in Table 3 in the total magnitude of the system raises them above the magnitude limit. All the observed multiple star systems have primary star $K$ magnitudes, obtained by combining the measured flux density ratios (Table 3 ) with the total flux densities (Table 1), that are brighter than the magnitude limit of the survey, and therefore do not appear to be biasing the measured $\mathrm{T}$ Tauri binary star frequency. The magnitude limit, however, does select for the more luminous and therefore younger portion of the $T$ Tauri star population given the vertical nature of the evolutionary tracks.

With this caveat, we assume that the difference in the binary star frequency between the $\mathrm{T}$ Tauri stars and their older counterparts on the main sequence is due to an intrinsic difference in their properties (i.e., the binary frequency depends on the age of the stars) and that the stars in the current $T$ Tauri star sample will have the same properties as the solar-type main-sequence sample once they evolve down to the main sequence.

If the $T$ Tauri stars and solar-type main-sequence stars have the same number of companion stars integrated over all possible separations, then the observed difference would be the result of a more peaked distribution for the $T$ Tauri stars. This would imply that the distribution of binary stars as a function of separation spreads or relaxes as a function of time. However, the binary star frequency for solar-type main-sequence stars integrated over all separations is 62 $[ \pm 6] \%$, which is comparable to the binary star frequency of $\mathrm{T}$ Tauri stars in the limited projected linear separation range 16 to $252 \mathrm{AU}$ and leaves only $2( \pm 11)$ companion stars to be found outside the separation range 16 to 252 $\mathrm{AU}$ if the total number of companions is to be conserved as a function of age. Tables 3 and 4 indicate that 16 compan- 
TABLE 9. Overlap of sample with different surveys.

\begin{tabular}{lccccc}
\hline \multicolumn{1}{c}{ Technique } & Ref & $\begin{array}{c}\text { Separation Range } \\
(\operatorname{arcsec})\end{array}$ & $\mathrm{N}_{\text {overlap }}$ & $\begin{array}{c}\mathrm{N}_{t} \\
\text { Triple Star Freq } \\
(\%)\end{array}$ & $\begin{array}{c}\text { pred } \\
\text { Direct Imaging }\end{array}$ \\
Lunar Occultation & 1,2 & $\geq 1.8-13$ & 27 & 4 & 15 \\
Spectroscopy & 1 & $0.005-0.1$ & 16 & 1 & 6 \\
Speckle & $3,4,5$ & $0.0002-0.003$ & 33 & 4 & 12 \\
All & 6 & $.1-1.8$ & 64 & - & 35 \\
\hline \hline
\end{tabular}

Notes: $\mathrm{N}_{\text {overlap }}$ is the number of overlap objects, i.e., the number of targets in the specified survey that are also in the complete sample of this work. $\mathrm{N}_{t}$ is the number of overlap objects that have companions observed both in the specified separation range and in the speckle imaging survey and are therefore triple or higher order systesms. The triple (or higher order) star frequency predicted is given by $\frac{\mathrm{N}_{t}}{\mathrm{~N}_{\text {overlap }}} \times 100 \%$. The last entry combines all the surveys to predict a triple (or higher order) star frequency of $35 \%$ for the $\mathrm{T}$ Tauri star sample.
(1) Simon et al. (1992)
(3) Mathieu et al. (1988)
(2) Simon (1992b)
(4) Mathieu (1992b)
(5) Edwards (1993)
(6) This work

ion stars have already been detected outside this separation range, even though the current knowledge of companion stars in the complete sample at all separations is far from complete. It therefore appears that the total number of companion stars is greater at the $\mathrm{T}$ Tauri stage of evolution than on the main sequence.

An overabundance of companion stars among the $T$ Tauri stars as compared to the solar-type main-sequence star requires that some of the current $T$ Tauri star pairs be disrupted by the time they evolve to the main sequence. The incomplete overlap of this sample with those observed with other techniques has already revealed that at least $14 \%$ of the target stars in this sample are members of systems with three or more components (see Tables 3 and 4), whereas Duquennoy \& Mayor (1991) find that only $5 \%$ of their solar-type main-sequence targets are members of these higher order systems. Furthermore, the incomplete overlap of only the various surveys, as opposed to isolated observations, done with other techniques predicts that $\sim 35 \%$ of the targets are triple or higher order systems with one component observable by speckle imaging (see Table 9). Thus one possible mechanism for reducing the number of star pairs in the separation range 16 to $252 \mathrm{AU}$ with increasing age is the disruption of young triple or higher order systems. The triples and quadruples that have been observed so far are all in hierarchical systems, i.e., the ratio of separations in the system is large $(\geqslant 10)$ and are therefore relatively stable in isolation, but could be "ionized" by close encounters with another star or system of stars. If disruption occurs, then the more widely separated stars (or pair of stars) would be more likely to break away. Thus one would expect to observe no difference between the $T$ Tauri and the main-sequence binary star frequencies at the shortest separation. This is consistent with current spectroscopic measurements of $\mathrm{T}$ Tauri stars, which reveal a binary star frequency at periods less than 100 days, or equivalently at the smallest separation, that is similar to that of the solar-type main-sequence stars (Mathieu et al. 1988; Mathieu 1992a). Thus the discrepancy between the number of companion stars observed for the $\mathrm{T}$ Tauri stars and the solar-type main-sequence stars may result from the disruption of some young triple and quadruple systems before they reach the main sequence.

\section{SUMMARY}

We have carried out a magnitude limited $(K<=8.5$ mag) multiplicity survey of $\mathrm{T}$ Tauri stars in the two nearest star forming regions the northern hemisphere, TauAur and Oph-Sco. Each of the 69 stars in the sample was imaged using two-dimensional speckle interferometric techniques. On average these observations were sensitive to binary stars with magnitude differences up to $3.1 \mathrm{mag}$ and separations ranging from 0 ". 1 to 1.8 . Thirty-three companion stars were found, of which nine are new detections.

There appears to be no statistically significant difference between the binary star frequency of Tau-Aur and OphSco. Similarly, the binary star frequency in the high mass cloud core $\rho$ Oph compared to that of the remainder of the sample shows no significant difference, although the small number of stars in $\rho$ Oph renders this comparison fairly inconclusive.

There is a distinction between the classical T Tauri stars (CTTS) and the weak-lined T Tauri stars (WTTS) based on the binary star frequency as a function of separation; the WTTS binary star distribution is enhanced at the smaller separations compared to the CTTS binary star distribution. The crossover separation occurs near $100 \mathrm{AU}$, the size typically quoted for a circumstellar disk. We suggest that all $\mathrm{T}$ Tauri stars begin as CTTS and become WTTS when accretion has ceased and that the nearby companion stars act to shorten the accretion time scale in multiple star systems.

Integrated over all magnitude differences the binary star frequency in the projected linear separation range 16 to 252 AU for T Tauri stars $(60[ \pm 17] \%)$ is a factor of 4 greater than that of the solar-type main-sequence stars (16 $[ \pm 3] \%)$. We propose that the observed overabundance of companions to $\mathrm{T}$ Tauri stars with respect to their older counterparts on the main sequence is an evolutionary effect; in this scheme triple and higher order $T$ Tauri systems, which are observed at higher frequencies than for the 
solar-type main-sequence stars, are ionized by close encounters with another star or system of stars.

We thank the staff of Palomar Observatory, especially the night assistants Juan Carrasco and Will McKinley, for their assistance during the observations and J. Graham, D. McCarthy, A. Sargent, M. Simon, and B. T. Soifer for many helpful discussions. We are grateful to S. Beckwith, P. Gorham, C. Haniff, C. Koresko, and S. Kulkarni for both shared telescope time and productive interactions and we are indebted to Fred Walter who kindly provided a target list of X-ray identified sources in the Oph-Sco regions prior to publication as well as many helpful comments in reviewing this paper. Tom Prince and the Caltech Conconcurrent Supercomputing Facility generously provided time on the Caltech NCUBE supercomputer for the speckle imaging data reduction. This research has made use of the SIMBAD data base, operated at CDS, Strasbourg, France. Infrared astrophysics at Caltech is supported by a grant from the NSF. A. G. currently receives support from NASA through Grant No. HF-1031.01-92A awarded by the Space Telescope Science Institute which is operated by the Association of Universities for Research in Astronomy, Inc., for NASA under Contract No. NAS526555.

\section{APPENDIX: THE BINARY STAR FREQUENCY OF MAIN-SEQUENCE STARS}

Duquennoy \& Mayor (1991) searched a total of 164 nearby solar-type main-sequence stars for companion stars and found that the number of companion stars as a function of the orbital period, $P$, could be fit by a Gaussian distribution in $\log P$. Figure 14(a) reproduces their binned, bias-corrected data as well as their model fit. To compare this work to the T Tauri star sample, their distribution is converted to a function of average projected linear separation $(\langle a\rangle)$. The period is converted to its corresponding semimajor axis, $A$, using Kepler's law and assuming an average primary star mass of $1 \mathscr{M}_{\odot}$ and an average mass ratio between the components of 0.40 (from Duquennoy and Mayor's Fig. 10). The semimajor axis is translated to an average projected separation based on a Monte-Carlo simulation carried out by Fischer \& Marcy (1992), which givers the following relationship,

$$
A=1.26\langle a\rangle \text {. }
$$

The distribution of solar-type main-sequence star companion stars as a function of $\langle a\rangle$ is then rebinned, using Duquennoy and Mayor's model fit. Figure 14(b) shows the result of rebinning the main-sequence data, such that the new bin width matches the separation range $\Delta \log \langle a\rangle_{A U}=1.2$, and normalizing the distribution by the total number of target stars (164) to produce a binary star
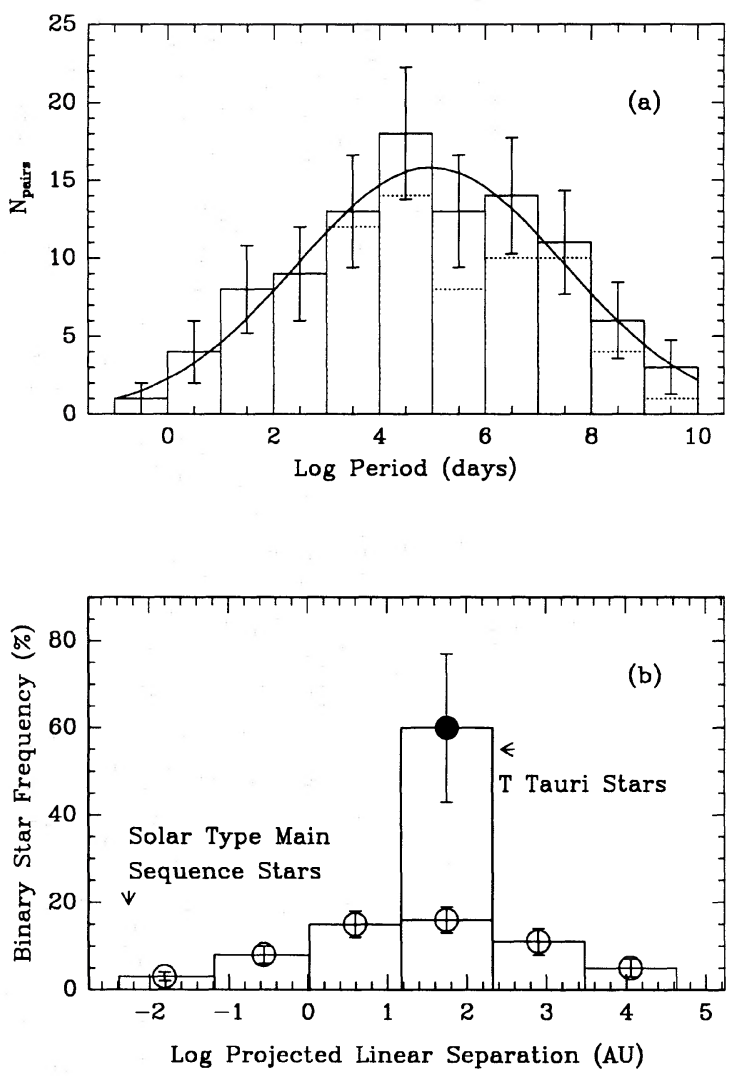

FIG. 14. (a) The distribution of companion stars in the nearby sample of solar-type main-sequence stars as a function of orbital period, taken from Duquennoy \& Mayor (1991) (b) The hatched histogram is the binary star frequency of the solar-type mainsequence stars as a function of average projected linear separation. Also shown is the binary star frequency of the $T$ Tauri stars (the unhatched region). The Tauri binary star frequency appears to be a factor of 4 greater than that of the solar-type main-sequence stars.

frequency distribution. Integrated over the projected linear separation range 16 to $252 \mathrm{AU}$, the binary star frequency of nearby solar-type main-sequence stars is $16[ \pm 3] \%$, where the uncertainty is based on Poisson statistics.

Fischer \& Marcy (1992) compile the results of several M dwarf surveys, conducted with different observing techniques, to determine the multiplicity of these low mass main-sequence stars. They quote their results in terms of the number of companion stars per target per AU for a given range of semimajor axes (their Table 2). Based on their relationship between $\langle a\rangle$ and $A$ to convert these binary star frequencies to a function of $\langle a\rangle$ and an integration of these values over 16 to $252 \mathrm{AU}$, the binary star frequency in this range for the $\mathbf{M}$ dwarfs is estimated to be $12[ \pm 4] \%$.

\section{REFERENCES}

Abt, H. A. 1983, ARA\&A, 21, 343

Abt, H. A., \& Levy, S. G 1976, ApJSS, 30, 273
Adams, F. C., Lada, C. J., \& Shu, F. H. 1988, ApJ, 326, 865

Aitken, R. F. 1932, in A New General Catalog of Double Stars Within 
$120^{\circ}$ of the North Pole (Carnegie Institution, Washington, D.C.)

Basri, G., \& Batalha, C. 1990, ApJ, 363, 654

Basri, G., \& Bertout, C. 1989, ApJ, 341, 340

Beckwith, S. V. W., \& Sargent, A. I. 1992, in Protostars and Planets III, edited by E. H. Levy and J. Lunine, preprint

Beckwith, S. V. W., Sargent, A. I., Chini, R. S., \& Gusten, R. 1990, AJ, 99, 924

Blaauw, A. 1964, ARA\&A, 2, 213

Bouvier, J., \& Appenzeller, I. 1991, A\&ASS, 92, 481

Bouvier, J., Tessier, E., \& Cabrit, S. 1992, A\&A, preprint

Cabrit, S., Edwards, S., Strom, S. E., \& Strom, K. M. 1990, ApJ, 354, 687

Chelli, A., Zinnecker, H., Carrasco, L., Cruz-Gonzalez, I., \& Perrier, C. 1988, A\&A, 207, 46

Chen, W. P., Simon, M., Longmore, A. J., Howell, R. R., \& Benson, J. A. 1990, ApJ, 357, 224

Christou, J. C., Cheng, A. Y. S., Hege, K. E., \& Roddier, C. 1985, AJ, 90, 2644

Cohen, M., Emerson, J. P., \& Beichman, C. A. 1989, ApJ, 339, 455

Cohen, M., \& Kuhi, L. V. 1979, ApJSS, 41, 743

de Geus, E. J., de Zeeuw, P. T., \& Lub, J. 1989, A\&A, 216, 44

de Geus, E. J., Bronfman, L., \& Thaddeus, P. 1990, A\&A, 231, 137

de Geus, E. J., \& Burton, W. B. 1991, A\&A, 246, 559

Duquennoy, A., \& Mayor, M. 1991, A\&A, 248, 485

Dyck, H. M., Simon, T., \& Zuckerman, B. 1982, ApJ, 255, L103

Edwards, S. 1993, private communication

Elias, J. H. 1978, ApJ, 224, 857

Fischer, D. A., \& March, G. W. 1992, ApJ, 396, 178

Ghez, A. M. 1992, Ph.D. thesis, California Institute of Technology

Ghez, A. M., Neugebauer, G., Gorham, P. W., Haniff, C. A., Kulkarni,

S. R., Matthews, K., Koresko, C., \& Beckwith, S. 1991, AJ, 102, 2066

Glass, I. S., \& Penston, M. V. 1974, MNRAS, 167, 237

Gorham, P. W., Ghez, A. M., Haniff, C. A., \& Prince, T. A. 1990, AJ, 100,294

Graham, J. 1991, private communication

Haas, M., Leinert, Ch., \& Zinnecker, H. 1990, A\&A, 230, L1

Haro, G., \& Chavira, E. 1974, Inf. Bull. Var. Stars, 926

Hartigan, P., Kenyon, S. J., Hartmann, L., Strom, S. E., Edwards, S.,

Welty, A. D., \& Stauffer, J. 1991, ApJ, 382, 617

Heintz, W. D. 1980, ApJSS, 44, 111

Henry, T. J. 1991, Ph.D. thesis, University of Arizona

Henry, T. J., McCarthy, D. W., Freeman, J., \& Christou, J. C. 1992, AJ, 103, 1369

Herbig, G. H. 1977, ApJ, 214, 747

Herbig, G. H., \& Bell, K. R. 1988, Lick Obs. Bull. No. 1111

Herbig, G. H., Vrba, F. J., \& Rydgren, A. E. 1986, AJ, 91, 575

Herbst, W., \& Warner, J. W. 1981, AJ, 86, 885

Joy, A. H., \& Van Biesbroeck, G. 1944, PASP, 56, 123

Koresko, C. D., Beckwith, S. V. W., Ghez, A. M., Matthews, K., \& Neugebauer, G. 1991, AJ, 102, 2073

Lada, E. A., Strom, K. M., \& Myers, P. C. 1992, in Protostars and

Planets III, edited by E. H. Levy and J. Lunine, preprint
Leinert, Ch., Haas, M., Richichi, A., Zinnecker, H., \& Mundt, R. 1991, A\&A, 250, 407

Leinert, Ch., et al. 1992, Complementary Approaches to Double and Multiple Star Research, IAU Colloquium No. 135, edited by H. A. McAlister and W. I. Hartkopf (ASP, San Francisco), p. 21

Lin, D. N. C., \& Papaloizou, J. C. B 1992, in Protostars and Planets III, edited by E. H. Levy and J. Lunine, preprint

Lynds, B. T. 1962, ApJSS, 7, 1

Mathieu, R. D., Walter, F. M., \& Myers, P. C. 1988, AJ, 98, 987

Mathieu, R. D. 1992a, Complementary Approaches to Double and Multiple Star Research, IAU Colloquium No. 135, edited by H. A. McAlister and W. I. Hartkopf (ASP, San Francisco), p. 30

Mathieu, R. D. 1992b, private communication

McAlister, H. A., \& Hartkopf, W. I. 1988, Second Catalog of Interferometric Measurements of Binary Stars (Center for High Angular Resolution Astronomy)

Moneti, A., \& Zinnecker, H. 1991, A \& A, 242, 428

Montmerle, T., Koch-Miramond, L., Falgarone, E., \& Grindlay, J. E. 1983, ApJ, 269, 182

Myers, P. C. 1985, in Protostars and Planets II, edited by D. C. Black and M. S. Mathews, p. 81

Rydgren, A. E., Schmelz, J. T., \& Vrba, F. J. 1982, ApJ, 256, 168

Rydgren, A. E., Strom, S. E., \& Strom, K. M. 1976, ApJSS, 30, 307

Rydgren, A. E., \& Vrba, F. J. 1981, AJ, 86, 1069

Rydgren, A. E., \& Vrba, F. J. 1983, AJ, 88, 1017

Shu, F. H., Adams, F. C., \& Lizano, S. 1987, ARA\&A, 25, 23

Simon, M. 1992a, Complementary Approaches to Double and Multiple Star Research, IAU Colloquium No. 135, edited by H. A. McAlister and W. I. Hartkopf (ASP, San Francisco), p. 41

Simon, M. 1992b, private communication

Simon, M., Chen, W. P., Howell, R. R., Benson, J. A., \& Slowik, D. 1992, ApJ, 384, 212

Simon, M., Howell, R. R., Longmore, A. J., Wilking, B. A., Peterson, D. M., \& Chen, W. P. 1987, ApJ, 320, 344

Skrutskie, M. F. 1992, private communication

Strom, K. M., Strom, S. E., Edwards, S., Cabrit, S., \& Skrutskie, M. F. 1989, AJ, 97, 1451

Ungerechts, H., \& Thaddeus, P. 1987, ApJSS, 63, 645

Walter, F. M. 1986, ApJ, 306, 573

Walter, F. M., Brown, A., Mathieu, R. D., Myers, P. C., \& Vrba, F. J. 1988, AJ, 96, 297

Walter, F. M., Vrba, F. J., Mathieu, R. D., Brown, A., \& Myers, P. C. 1993, in preparation

Warner, G., Strom, S. E., \& Strom, K. M. 1977, ApJ, 213, 427

Weintraub, D. 1989, Ph.D. thesis, University of California, Los Angeles Wilking, B. A., \& Lada, C. J. 1983, ApJ, 274, 698

Wilking, B. A., Lada, C. J., \& Young, E. T. 1989, ApJ, 340, 823

Zinnecker, H., Brandner, W., Reipurth, B. 1992, Complementary Approaches to Double and Multiple Star Research, IAU Colloquium No. 135, edited by H. A. McAlister and W. I. Hartkopf (ASP, San Fran(isco), p. 50 